TRANSACTIONS OF THE

AMERICAN MATHEMATICAL SOCIETY

Volume 361, Number 9, September 2009, Pages 4999-5021

S 0002-9947(09)04716-3

Article electronically published on April 15, 2009

\title{
A CONSTRUCTION OF NUMERICAL CAMPEDELLI SURFACES WITH TORSION $\mathbb{Z} / 6$
}

\author{
JORGE NEVES AND STAVROS ARGYRIOS PAPADAKIS
}

\begin{abstract}
We produce a family of numerical Campedelli surfaces with $\mathbb{Z} / 6$ torsion by constructing the canonical ring of the étale 6 to 1 cover using serial unprojection. In Section 2 we develop the necessary algebraic machinery. Section 3 contains the numerical Campedelli surface construction, while Section 4 contains remarks and open questions.
\end{abstract}

\section{INTRODUCTION}

A numerical Campedelli surface is a smooth minimal surface of general type over the complex numbers with $K^{2}=2$ and $q=p_{g}=0$. It is known that the algebraic fundamental group $\pi_{1}^{\text {alg }}$ of such a surface is finite, of order at most 9 (cf. BPHV] Chap. VII.10). Two recent papers about numerical Campedelli surfaces are MP and $[\mathrm{LP}$; the first classifies the case where the algebraic fundamental group has order exactly 9 , while the second gives simply connected examples.

In the present work we give a construction of numerical Campedelli surfaces with algebraic fundamental group equal to $\mathbb{Z} / 6$. To our knowledge, there were no such examples previously known, and it settles the existence question for numerical Campedelli surfaces with algebraic fundamental group of order 6 , since by $\mathrm{Na}$ there are no numerical Campedelli surfaces with algebraic fundamental group equal to the symmetric group of order 6 .

Our approach is to construct, using serial unprojection of type Kustin-Miller, the canonical ring of the étale 6 to 1 cover together with a suitable basepoint free action of $\mathbb{Z} / 6$. The cover is a regular canonical surface with $p_{g}=5$, and $K^{2}=12$, canonically embedded in $\mathbb{P}\left(1^{5}, 2^{3}\right)$.

The number of moduli of our construction is at most 4; cf. Remark 3.17 below. Since the expected number of moduli of a numerical Campedelli is 6 (cf. BPHV), p. 295), it follows that there exist numerical Campedelli surfaces with torsion $\mathbb{Z} / 6$ that cannot be obtained by our construction.

In Section 2 we define, for $n \geq 2$, what we call the generic $\left(\begin{array}{l}n \\ 2\end{array}\right)$ Pfaffians ideal (Definition 2.2) and prove that it is Gorenstein of codimension equal to $n+1$ (Theorem 2.3). A special case of the construction is due to Frantzen ([Fr, Section 2.4).

In Section 3 we apply the results of the previous sections in the case of $n=4$ to our specific geometric situation. The main results are Theorems 3.12 and 3.16 , where we settle the existence of a nonsingular regular surface with $p_{g}=5$ and

Received by the editors April 13, 2007 and, in revised form, December 3, 2007.

2000 Mathematics Subject Classification. Primary 14J29; Secondary 13H10, 14M05.

(C)2009 American Mathematical Society

Reverts to public domain 28 years from publication 
$K^{2}=12$ endowed with a $\mathbb{Z} / 6$ basepoint free action. Finally, Section 4 contains some remarks and open questions.

The way in which we have arrived at the family constructed in this article is strongly influenced by the general theory in [R1]. More precisely, one assumes that a hypothetical étale 6 to 1 cover of a numerical Campedelli surface is a quadratic section of an anticanonically embedded Fano 3 -fold $V$, as in many similar constructions of surfaces of general type. Then, standard numerical Hilbert series calculations (cf. [R1], Section 3) lead to the expectation that the anticanonical model of $V$ is a codimension 5 projectively Gorenstein subscheme of $\mathbb{P}\left(1^{5}, 2^{4}\right)$ with a certain Hilbert series. Combining this with the knowledge of how the Hilbert series changes during unprojection (or, alternatively, and more easily, reading the result directly from $[\mathrm{Br}]$ ) one concludes that $V$ can be realized as the result of a series of 4 unprojections of Kustin-Miller type, starting from a degree 4 hypersurface in $\mathbb{P}\left(1^{5}\right)$. Hence, starting from a degree 4 hypersurface in $\mathbb{P}\left(1^{5}\right)$ and unprojecting an arrangement of 4 codimension 1 loci, one could obtain a 3-fold $V$ in $\mathbb{P}\left(1^{5}, 2^{4}\right)$ with the right Hilbert series. Then, taking a suitable member of $\left|-2 K_{V}\right|$ we would obtain the étale 6 to 1 cover of a numerical Campedelli.

However, to set up a free $\mathbb{Z} / 6$ action, motivated by empirical evidence showing that unprojection is best calculated in a general framework, we were driven to the unprojection of a general set of 4 linear subspaces of dimension 5 in a degree 46 -fold hypersurface in $\mathbb{P}\left(1^{8}\right)$. Our main motivation was that we could then assume that these loci were defined by $x_{1}=x_{2}=0, x_{3}=x_{4}=0$, etc. After the unprojection of these subspaces we obtained a 6 -fold in $\mathbb{P}\left(1^{8}, 2^{4}\right)$, and then taking 3 linear sections and 1 quadratic section we constructed a family of surfaces with $p_{g}=5, q=0, K^{2}=$ 12. Afterwards, calculations with characters and $G$-Hilbert series helped us to discover a suitable subfamily endowed with a good $\mathbb{Z} / 6$ action.

We believe that a similar approach could be useful to other situations as well; compare Remark 4.3 below.

Bearing in mind the expectation that under mild conditions unprojections commute (cf. Remark 2.6 below) and the previously done calculations of $\mathrm{P} 2$ and $\mathrm{Fr}$, Section 2.4, we obtained the generic $\left(\begin{array}{l}n \\ 2\end{array}\right)$ Pfaffians ideal format for $n=4$. We then discovered that the arguments for the Gorensteinness of the format for general $n$ were a rather straightforward generalisation of those needed in the special case.

\section{The generic $\left(\begin{array}{l}n \\ 2\end{array}\right)$ Pfaffians ideal}

Notation 2.1. Let us make the following notation:

(1) Let $n \geq 2$. Let $A_{0}=\mathbb{Z}\left[x_{1}, \ldots, x_{n}, z_{1}, \ldots, z_{n}, r_{d_{1} \cdots d_{n}}\right]$ be the polynomial ring over the integers in $n$ variables $x_{1}, \ldots, x_{n}, n$ variables $z_{1}, \ldots, z_{n}$ and $2^{n}$ variables $r_{d_{1} \cdots d_{n}}$, indexed by $\left(d_{1}, \ldots, d_{n}\right) \in\{0,1\}^{n}$.

(2) Define the polynomial algebra extensions $A_{0} \subset A_{1} \subset A_{2} \subset \cdots \subset A_{n}$ by setting inductively $A_{i}=A_{i-1}\left[y_{i}\right]$ for $i=1, \ldots, n$.

(3) Make these rings graded by setting the degree of $x_{i}, z_{i}$ and $r_{d_{1} \cdots d_{n}}$ equal to 1 , for all $i=1, \ldots, n$ and $\left(d_{1}, \ldots, d_{n}\right) \in\{0,1\}^{n}$ and by setting the degree of $y_{i}$ equal to $n-1$, for all $i=1, \ldots, n$.

(4) Consider the degree $n+1$ homogeneous polynomial defined by

$$
Q=\sum r_{d_{1} \cdots d_{n}} a_{1, d_{1}} \cdots a_{n, d_{n}} \in A_{0},
$$


where the summation is for $\left(d_{1}, \ldots, d_{n}\right) \in\{0,1\}^{n}$, and, by definition, $a_{i, d_{i}}$ is equal to $x_{i}$ if $d_{i}=0$ and equal to $z_{i}$ if $d_{i}=1$.

(5) For each $1 \leq i<j \leq n$, let

$Q_{i j}^{x x}=\frac{\partial^{2} Q}{\partial x_{i} \partial x_{j}}, \quad Q_{i j}^{x z}=\frac{\partial^{2} Q}{\partial x_{i} \partial z_{j}}, \quad Q_{i j}^{z x}=\frac{\partial^{2} Q}{\partial z_{i} \partial x_{j}} \quad$ and $\quad Q_{i j}^{z z}=\frac{\partial^{2} Q}{\partial z_{i} \partial z_{j}}$.

Then each of the $Q_{i j}^{a b}$, where $a, b \in\{x, y\}$, is homogeneous of degree $n-1$, and clearly,

$$
Q=x_{i} x_{j} Q_{i j}^{x x}+x_{i} z_{j} Q_{i j}^{x z}+z_{i} x_{j} Q_{i j}^{z x}+z_{i} z_{j} Q_{i j}^{z z} .
$$

(6) For each $1 \leq i<j \leq n$ let

$$
M_{i j}=\left(\begin{array}{ccccc}
0 & x_{i} & z_{i} & -x_{j} & -z_{j} \\
& 0 & y_{j} & Q_{i j}^{z z} & -Q_{i j}^{z x} \\
& & 0 & -Q_{i j}^{x z} & Q_{i j}^{x x} \\
& - \text { sym } & & 0 & -y_{i} \\
& & & & 0
\end{array}\right)
$$

be a skew-symmetric $5 \times 5$ matrix with entries in $A_{n}$. The 5 submaximal Pfaffians 1 of this matrix are:

$$
\begin{aligned}
& y_{i} y_{j}-Q_{i j}^{x z} Q_{i j}^{z x}+Q_{i j}^{x x} Q_{i j}^{z z}, \\
& x_{i} y_{i}+\left(x_{j} Q_{i j}^{z x}+z_{j} Q_{i j}^{z z}\right), \quad z_{i} y_{i}-\left(x_{j} Q_{i j}^{x x}+z_{j} Q_{i j}^{x z}\right), \\
& x_{j} y_{j}+\left(x_{i} Q_{i j}^{x z}+z_{i} Q_{i j}^{z z}\right), \quad z_{j} y_{j}-\left(x_{i} Q_{i j}^{x x}+z_{i} Q_{i j}^{z x}\right),
\end{aligned}
$$

which are all homogeneous elements of $A_{n}$. Fixing $1 \leq i \leq n$ and varying $j$ we see that several Pfaffians involve the monomial $x_{i} y_{i}$. Notice, however, that $x_{j} Q_{i j}^{z x}+z_{j} Q_{i j}^{z z}=\frac{\partial Q}{\partial z_{i}}$, which does not depend on $j$. Hence, in the set of Pfaffians of all possible $M_{i j}$, there is only 1 polynomial in which the monomial $x_{i} y_{i}$ occurs. A similar observation applies to the Pfaffians in which the monomial $z_{i} y_{i}$ occurs.

(7) For each $0 \leq p \leq n$ we define an homogeneous ideal $I_{p} \subset A_{p}$ by:

(a) $I_{0}=(Q) \subset A_{0}$;

(b) $I_{1}=\left(x_{1} y_{1}+\frac{\partial Q}{\partial z_{1}}, z_{1} y_{1}-\frac{\partial Q}{\partial x_{1}}\right) \subset A_{1}$, the ideal of $A_{1}$ generated by the two Pfaffians of $M_{12}$ which involve $x_{1} y_{1}$ and $z_{1} y_{1}$;

(c) for $2 \leq p \leq n, I_{p} \subset A_{p}$ is the ideal of $A_{p}$ generated by the union of all the submaximal Pfaffians of all matrices $M_{i j}$ for $1 \leq i<j \leq p$.

(8) In the set of Pfaffians of all possible $M_{a b}, 1 \leq a<b \leq n$, denote by

(a) $l_{i}$ the polynomial in which $x_{i} y_{i}$ occurs with coefficient 1 ;

(b) $m_{i}$ the polynomial in which $z_{i} y_{i}$ occurs with coefficient 1 ;

(c) $e_{i j}$ the polynomial in which $y_{i} y_{j}$ occurs with coefficient 1

for all $1 \leq i \leq n$ in (a) and (b), and $1 \leq i<j \leq n$ in (c). In particular,

$$
I_{p}=\left(\left\{e_{i j}, l_{t}, m_{t}: 1 \leq i<j \leq p \text { and } 1 \leq t \leq p\right\}\right) \subset A_{p},
$$

and in addition $I_{1}=\left(l_{1}, m_{1}\right) \subset A_{1}$. Let us stress that we can take the expressions in (2.1) for any given $j$ to write the polynomials $l_{i}, m_{i}$ and $e_{i j}$.

(9) For $0 \leq p \leq n-1$, define the homogeneous ideals $J_{p} \subset A_{p}$ as follows:

(a) $J_{0}=\left(x_{1}, z_{1}\right) \subset A_{0}$;

(b) $J_{p}=\left(x_{p+1}, z_{p+1}, y_{1}, \ldots, y_{p}\right) \subset A_{p}$, for $p \geq 1$.

${ }^{1}$ For a discussion about the Pfaffians of a skew-symmetric matrix see, for example, $[\mathrm{BH}]$, Section 3.4. 
(10) Finally, notice that $I_{p} \subset J_{p} \subset A_{p}$. Set $R_{p}=A_{p} / I_{p}$ and consider $J_{p}$ as an ideal of $R_{p}$.

Definition 2.2. We call the ideal $I_{n}$ of $A_{n}$ the generic $\left(\begin{array}{l}n \\ 2\end{array}\right)$ Pfaffians ideal.

The main aim of this section is to prove by induction on $p=0,1, \ldots, n$ that $R_{p}=$ $A_{p} / I_{p}$ is a Gorenstein graded ring whose dimension is equal to $\operatorname{dim} A_{0}-1$ (hence $I_{p}$ has codimension $p+1$ in $A_{p}$ ). Our strategy is to establish inductively that $R_{p}$ is the result of serial unprojection of type Kustin-Miller ( $[\mathrm{PR}$, Definition 1.2). Our main algebraic result is the following theorem, which we will prove in Subsection 2.1.

Theorem 2.3. Let all the notation be as above.

(a) For $p=1, \ldots, n, R_{p}$ is the unprojection ring of type Kustin-Miller of the pair $J_{p-1} \subset R_{p-1}$.

(b) For $p=0,1, \ldots, n, R_{p}$ is a normal Gorenstein graded integral domain of dimension equal to $\operatorname{dim} R_{0}$ (which is equal to $2 n+2^{n}$ since $\operatorname{dim} \mathbb{Z}=1$ ).

(c) There are natural inclusions

$$
R_{0} \subset R_{1} \subset \cdots \subset R_{p} \subset K\left(R_{0}\right),
$$

where $K\left(R_{0}\right)$ is the field of fractions of $R_{0}$, all except the last induced by the chain of inclusions $A_{0} \subset A_{1} \subset \cdots \subset A_{p}$.

(d) For $p=0, \ldots, n$ there exists a Zariski closed subset $F_{p} \subset \operatorname{Spec} R_{p}$, with the codimension of $F_{p}$ in $\operatorname{Spec} R_{p}$ at least 2, such that the open subscheme Spec $R_{p} \backslash F_{p}$ is naturally isomorphic with an open subscheme of Spec $R_{0}$.

(e) For $p=1, \ldots, n, x_{p}, z_{p}$ is a regular sequence of $R_{p}$.

(f) For $p=0,1, \ldots, n$ and $1 \leq i<j \leq n, x_{i}, x_{j}$ is a regular sequence of $R_{p}$.

Remark 2.4. The most important conclusions of Theorem 2.3 are (a) and (b). However, for the purposes of the inductive step we need all six statements.

Remark 2.5. For $1 \leq t \leq n$ the inclusion $R_{t-1} \subset R_{t}$ of (c) of Theorem 2.3 is given by $R_{t}=R_{t-1}\left[s_{t}\right]$, where $s_{t} \in K\left(R_{0}\right)$ is the rational function given by

$$
s_{t}=\frac{x_{t} y_{t}-l_{t}}{x_{t}}=\frac{z_{t} y_{t}-m_{t}}{z_{t}}
$$

and

for $1 \leq i \leq t-1$.

$$
s_{t}=\frac{y_{i} y_{t}-e_{i t}}{y_{i}}
$$

Remark 2.6. Fix $2 \leq p \leq n$. Inside Spec $R_{0}$ we have the $p$ codimension 1 subschemes $V\left(x_{i}, z_{i}\right)$ for $1 \leq i \leq p$. We can interpret Theorem 2.3 and Corollary 2.13 below as saying that the order we perform the unprojections of the subschemes is irrelevant. An interesting open question is to find general conditions that will guarantee this kind of commutativity of unprojections.

2.1. Proof of Theorem 2.3. We begin the proof of Theorem 2.3, The following proposition will be used in the proof of Proposition 2.8

Proposition 2.7. Denote by $\mathbb{Z}[X]$ the polynomial ring $\mathbb{Z}\left[x_{1}, \ldots, x_{n}\right]$, and assume $I \subset \mathbb{Z}[X]$ is a proper ideal generated by monic monomials. For any field $k$ we have

$$
\operatorname{dim} \frac{\mathbb{Z}[X]}{I} \otimes_{\mathbb{Z}} k=\operatorname{dim} \frac{\mathbb{Z}[X]}{I}-1 .
$$


Proof. Let $k$ be a field. Denote, for $d \geq 1$, by $\widetilde{S_{d}}$ the set of monic monomials of $\mathbb{Z}[X]$ of degree $d$ which are not in $I$. Since $I$ is generated by monic monomials, it follows that $\widetilde{S_{d}}$ is a $k$-basis of the degree $d$ homogeneous component of $\mathbb{Z}[X] / I \otimes_{\mathbb{Z}} k$. As a consequence, the Hilbert polynomial of $\mathbb{Z}[X] / I \otimes_{\mathbb{Z}} k$ is independent of the choice of the field $k$. Therefore, by [Ei], Corollary 13.7 the dimension of $\mathbb{Z}[X] / I \otimes_{\mathbb{Z}} k$ is also independent of the choice of the field $k$.

Being a free $\mathbb{Z}$-module, the polynomial ring $\mathbb{Z}[X]$ is a faithfully flat $\mathbb{Z}$-module. Moreover, the height of a homogeneous ideal of a graded Cohen-Macaulay ring is equal to its codimension; $\mathrm{cf}$. $[\mathrm{BH}$, Corollary 2.1.4. The result now follows from [BV], Proposition 3.14 (for $J=0$ ), taking into account that $\operatorname{dim} \mathbb{Z}[X]=\operatorname{dim} k[X]+1$.

Proposition 2.8. Fix $1 \leq p \leq n$. Assume $R_{p}$ is Cohen-Macaulay with $\operatorname{dim} R_{p}=$ $\operatorname{dim} R_{0}$. Then $x_{p}, z_{p}$ is a regular sequence for $R_{p}$.

Proof. Denote by $T \subset A_{p}$ the ideal of $A_{p}$ generated by all $r_{d_{1} \cdots d_{n}}$ for $\left(d_{1}, \ldots, d_{n}\right) \in$ $\{0,1\}^{n}$ with $\left(d_{1}, \ldots, d_{n}\right) \neq(0,0, \ldots, 0)$ and $\left(d_{1}, \ldots, d_{n}\right) \neq(1,1, \ldots, 1)$.

Using the assumptions about the dimension and the Cohen-Macaulayness of $R_{p}$, to prove the proposition it is enough to show that

$$
\operatorname{dim} R_{p} /\left(x_{p}, z_{p}\right) \leq \operatorname{dim} R_{0}-2=\operatorname{dim} A_{0}-3,
$$

and for that it is enough to show that

$$
\operatorname{dim} A_{p} /\left(I_{p}+\left(x_{p}, z_{p}, y_{p}\right)+T\right) \leq\left(\operatorname{dim} A_{0}-3\right)-\left(2^{n}-2\right)-1=2 n-1 .
$$

We denote by $\eta\left(l_{i}\right)$ the polynomial obtained from $l_{i}$ by setting the variables $x_{p}, z_{p}, y_{p}$ and $r_{d_{1} \cdots d_{n}}$ for $\left(d_{1}, \ldots, d_{n}\right) \in\{0,1\}^{n}$ with $\left(d_{1}, \ldots, d_{n}\right) \neq(0,0, \ldots, 0)$ and $\left(d_{1}, \ldots, d_{n}\right)$ $\neq(1,1, \ldots, 1)$ equal to 0 , and similarly for $\eta\left(m_{i}\right)$ and $\eta\left(e_{i j}\right)$.

For $1 \leq i<p$ we have

$$
\eta\left(l_{i}\right)=x_{i} y_{i}, \quad \eta\left(m_{i}\right)=z_{i} y_{i},
$$

for $1 \leq i<j<p$ we have

$$
\eta\left(e_{i j}\right)=y_{i} y_{j}
$$

and for $1 \leq i<p$ we have

$$
\begin{aligned}
& \eta\left(e_{i p}\right)=r_{00 \cdots 0} r_{11 \cdots 1}\left[\prod_{t=1}^{i-1} x_{t} z_{t}\right]\left[\prod_{t=i+1}^{p-1} x_{t} z_{t}\right]\left[\prod_{t=p+1}^{n} x_{t} z_{t}\right], \\
& \eta\left(l_{p}\right)=r_{11 \cdots 1}\left[\prod_{t=1}^{p-1} z_{t}\right]\left[\prod_{t=p+1}^{n} z_{t}\right] \\
& \eta\left(m_{p}\right)=-r_{00 \cdots 0}\left[\prod_{t=1}^{p-1} x_{t}\right]\left[\prod_{t=p+1}^{n} x_{t}\right] .
\end{aligned}
$$

For the proof of the first equality, substitute $x_{p}=z_{p}=0$ to

$$
l_{i}=x_{i} y_{i}+x_{p} \frac{\partial^{2} Q^{\prime}}{\partial z_{i} \partial x_{p}}+z_{p} \frac{\partial^{2} Q^{\prime}}{\partial z_{i} \partial z_{p}}
$$

where $Q^{\prime}=r_{00 \cdots 0} x_{1} \cdots x_{n}+r_{11 \cdots 1} z_{1} \cdots z_{n}$. The proof of the other equalities is similar. 
To calculate the codimension of the ideal $\left(I_{p}+\left(x_{p}, z_{p}, y_{p}\right)+T\right)$ of $A_{p}$ using Proposition 2.7, we can make a base change from $\mathbb{Z}$ to an algebraically closed field $k$ and argue geometrically by studying zero loci. Since

$$
I_{p}=\left(l_{1}, \ldots, l_{p}, m_{1}, \ldots, m_{p}, e_{i j}\right) \subset A_{p}
$$

(with indices $1 \leq i<j \leq p$ ), using the vanishing of $\eta\left(e_{i j}\right)=y_{i} y_{j}$, for $1 \leq i<j<p$, we get two cases.

Case 1. All $y_{i}$ are 0 , for $1 \leq i \leq p-1$. Then, the vanishing of $\eta\left(l_{p}\right)$ and $\eta\left(m_{p}\right)$ imply that two more variables vanish, so we get the desired codimension.

Case 2. There exists unique nonzero $y_{a}$, with $1 \leq a \leq p-1$. Using the vanishing of $\eta\left(l_{a}\right)$ and $\eta\left(m_{a}\right)$ we get the vanishing of both $x_{a}$ and $z_{a}$, and using the vanishing of $\eta\left(e_{a p}\right)$ we get that at least 1 more variable should vanish, so we again reach the desired codimension which finishes the proof of the proposition.

We now start the induction for the proof of Theorem 2.3.

Lemma 2.9. Theorem 2.3 is true for $p=0$.

Proof. The ring $A_{0}$ is a Gorenstein normal integral domain, since it is a finitely generated polynomial $\mathbb{Z}$-algebra. Since $Q \in A_{0}$ is an irreducible polynomial, it follows that $R_{0}=A_{0} /(Q)$ is a Gorenstein integral domain. Therefore, using the fact that $m$ homogeneous elements (of positive degree) of a graded Cohen-Macaulay ring form a regular sequence if and only if the ideal they generate has codimension $m$ (cf. [BH], Theorem 2.1.2), to prove that, for fixed $1 \leq i<j \leq n, x_{i}, x_{j}$ is a regular sequence of $R_{0}$, it is enough to show that $\operatorname{dim} R_{0} /\left(x_{i}, x_{j}\right)=\operatorname{dim} R_{0}-2$. This is true, since $R_{0} /\left(x_{i}, x_{j}\right)$ is isomorphic to the quotient of a polynomial ring over the integers in two less variables than $A_{0}$ by the ideal generated by a nonzero nonconstant homogeneous polynomial.

We will prove the normality of $R_{0}$ by applying [BV], Lemma 16.24, which says that a Noetherian ring $R$ is normal if there exists $y \in R$ which is not a zero-divisor, such that $R /(y)$ is reduced and $R\left[y^{-1}\right]$ is normal.

Fix $1 \leq i \leq n$. The ring $R_{0} /\left(x_{i}\right)$ is a reduced ring, since the polynomial obtained from $Q$ by substituting 0 for $x_{i}$ has no multiple factors.

Denote, for $1 \leq i \leq n$, by $T_{i} \subset R_{0}$ the multiplicatively closed subset

$$
T_{i}=\left\{1, x_{i}, x_{i}^{2}, x_{i}^{3}, \ldots\right\} \subset R_{0} .
$$

For notational convenience we also set $T_{0}=\{1\} \subset R_{0}$. We will prove by induction on $t=0,1, \ldots, n$ that the localization ring

$$
B_{t}=T_{n-t}^{-1} T_{n-t-1}^{-1} \cdots T_{1}^{-1} T_{0}^{-1} R_{0}
$$

is a normal integral domain. Since a localization of an integral domain is an integral domain, we only need to prove the normality of $B_{t}$.

Assume first that $t=0$. By the form of $Q, B_{0}$ is isomorphic to a localization of the polynomial subring

$$
\mathbb{Z}\left[x_{1}, \ldots, x_{n}, z_{1}, \ldots, z_{n}, r_{d_{1} \cdots d_{n}}\right] \subset A_{0},
$$

where $\left(d_{1}, d_{2}, \ldots, d_{n}\right) \in\{0,1\}^{n}$ and $\left(d_{1}, \ldots, d_{n}\right) \neq(0,0, \ldots, 0)$. Since the localization of a normal ring is again normal, we get that $B_{0}$ is a normal domain.

Assume now that for some $t$ with $0 \leq t \leq n-1$ we have that $B_{t}$ is normal. By [BV], Lemma 16.24, to prove that the domain $B_{t+1}$ is normal it is enough to prove 
that $B_{t+1} /\left(x_{n-t}\right)$ is reduced. Since localization commutes with taking quotients and the localization of a reduced ring is again reduced, we have that $B_{t+1} /\left(x_{n-t}\right)$ is reduced as a localization of the already proven reduced $R_{0} /\left(x_{n-t}\right)$. This finishes the induction, and hence the case $p=0$ of Theorem 2.3 follows.

Lemma 2.10. Theorem 2.3 is true for $p=1$.

Proof. Using [P2, Section 4, we get that $R_{1}$ is the unprojection of type KustinMiller of the pair $J_{0} \subset R_{0}$; hence by the definitions of unprojection ([PR], Section 1) we have that $R_{0}$ is contained in a natural way in $R_{1}$ and $R_{1}$ has the same dimension as $R_{0}$. Moreover, by $\left[\mathrm{PR}\right.$, Theorem 1.5, $R_{1}$ is Gorenstein, and by $[\mathrm{PR}$, Remark 1.5, $R_{1}$ is a domain contained in a natural way in the field of fractions $K\left(R_{0}\right)$ of $R_{0}$. In particular, the ring $R_{1}$ is Cohen-Macaulay and hence satisfies Serre's conditions $S_{i}$ for all $i \geq 0$ (cf. $[\mathrm{BH}]$, p. 63).

Proposition 2.8 says that $x_{1}, z_{1}$ is a regular sequence of $R_{1}$. Hence by setting

$$
F_{1}=V\left(x_{1}, z_{1}\right) \subset \operatorname{Spec} R_{1}
$$

we get that $F_{1}$ has codimension 2 in Spec $R_{1}$, and by the construction of unprojection Spec $R_{1} \backslash F_{1}$ is isomorphic in a natural way (induced by the inclusion $R_{0} \subset R_{1}$ ) to the open subscheme Spec $R_{0} \backslash V\left(x_{1}, z_{1}\right)$ of Spec $R_{0}$. Using Serre's normality criterion ( $\left[\mathrm{BH}\right.$, Theorem 2.2.22), we get that the integral domain $R_{1}$ is normal.

We now prove that if $1 \leq i<j \leq n$, then $x_{i}, x_{j}$ is a regular sequence of $R_{1}$. Assume this is not true. Using the fact that we proved that $R_{1}$ is Gorenstein and that $m$ homogeneous elements (of positive degree) of a graded Cohen-Macaulay ring form a regular sequence if and only if the ideal they generate has codimension $m$ (cf. $\left[\mathrm{BH}\right.$, Theorem 2.1.2), we get that $V\left(x_{i}, x_{j}\right) \subset$ Spec $R_{1}$ has codimension at most 1 in $\operatorname{Spec} R_{1}$. Using the natural isomorphism of $\operatorname{Spec} R_{1} \backslash F_{1}$ with the open subscheme $\operatorname{Spec} R_{0} \backslash V\left(x_{1}, z_{1}\right)$ and the fact that we proved that $F_{1} \subset \operatorname{Spec} R_{1}$ has codimension 2 in $\operatorname{Spec} R_{1}$, it follows that $x_{i}, x_{j}$ is not a regular sequence for $R_{0}$, contradicting Lemma 2.9. This finishes the proof of Theorem 2.3 for $p=1$.

We now do the inductive step in the proof of Theorem 2.3. We fix $q$ with $1 \leq q \leq n-1$. We assume that Theorem 2.3 is true for all values $p$ with $0 \leq p \leq q$, and we will show that it is also true for the case $p=q+1$.

For the rest of the proof, given $0 \leq t \leq n-1$, we will denote by $L_{t} \subset R_{0}$ the ideal $L_{t}=\left(x_{t+1}, z_{t+1}\right) \subset R_{0}$, and by $i_{t}: J_{t} \rightarrow R_{t}$ and $i_{1, t}: L_{t} \rightarrow R_{0}$ the natural inclusion homomorphisms.

Lemma 2.11. There exists a unique homomorphism of Abelian groups

$$
\phi_{q}: \operatorname{Hom}_{R_{0}}\left(L_{q}, R_{0}\right) \rightarrow \operatorname{Hom}_{R_{q}}\left(J_{q}, R_{q}\right)
$$

such that

$$
\phi_{q}(f)\left(x_{q+1}\right)=f\left(x_{q+1}\right)
$$

for all $f \in \operatorname{Hom}_{R_{0}}\left(L_{q}, R_{0}\right)$.

Proof. Recall that if $L \subset R$ is an ideal of a commutative ring $R$ and $w \in L$ a nonzero divisor of $R$, then $\operatorname{Hom}_{R}(L, R)$ is isomorphic to the ideal $\{a \in R: a L \subset(w)\}$ by the map $f \mapsto f(w)$ (cf. [PR, Remark 1.3.3). In particular, $f$ is uniquely specified by the value $f(w)$. 
Accordingly, since by the inductive hypothesis both $R_{0}$ and $R_{q}$ are integral domains with $R_{0} \subset R_{q}$, it is enough to show that

$$
\left\{a \in R_{0}: a L_{q} \subset R_{0} x_{q+1}\right\} \subset\left\{b \in R_{q}: b J_{q} \subset R_{q} x_{q+1}\right\} .
$$

Suppose $a \in R_{0}$ and $a L_{q} \subset R_{0} x_{q+1}$. In particular, $a z_{q+1} \in R_{0} x_{q+1}$. Obviously $a \in R_{q}$, so we need to show that $a y_{i} \subset R_{q} x_{q+1}$ for all $1 \leq i \leq q$.

Fix $1 \leq i \leq q$. Using the equation $l_{i}$, which is 0 at $R_{q}$, we get

$$
x_{i} a y_{i}=-\left(a x_{q+1} Q_{i, q+1}^{z x}+a z_{q+1} Q_{i, q+1}^{z z}\right) \in R_{q} x_{q+1} .
$$

By the inductive hypothesis, $x_{i}, x_{q+1}$ is a regular sequence for $R_{q}$. As a consequence, (2.2) implies that $a y_{i} \in R_{q} x_{q+1}$. Hence we get the existence of the map $\phi_{q} . R_{q}$ is a domain which implies that $x_{q+1}$ is a regular element of $R_{q}$. The uniqueness follows by the fact that an element of $\operatorname{Hom}_{R_{q}}\left(J_{q}, R_{q}\right)$ is uniquely specified by its value at $x_{q+1}$.

Notice that clearly $\phi_{q}\left(i_{1, q}\right)=i_{q}$.

Proposition 2.12. Assume $f \in \operatorname{Hom}_{R_{q}}\left(J_{q}, R_{q}\right)$. There exists $b \in R_{q}$ such that the homomorphism $f-b i_{q}$ maps $x_{q+1}$ and $z_{q+1}$ inside $R_{0} \subset R_{q}$.

Proof. We prove by induction that for every $t=0, \ldots, q$ there exists $b_{t} \in R_{q}$ such that $f-b_{t} i_{q}$ maps the elements $x_{q+1}, z_{q+1}$ and $y_{j}$, for $1 \leq j \leq q-t$, inside $R_{q-t} \subset R_{q}$.

The result is trivially true when $t=0$. Assume $0 \leq t \leq q-1$ and that there exists $b_{t} \in R_{q}$ such that $f-b_{t} i_{q}$ maps the elements $x_{q+1}, z_{q+1}$ and $y_{j}$, for $1 \leq j \leq q-t$, inside $R_{q-t}$. Since, by construction, $R_{q-t}=R_{q-(t+1)}\left[y_{q-t}\right]$ (as algebras), there exist $a \in R_{q-(t+1)}$ and $c \in R_{q}$ with

$$
\left(f-b_{t} i_{q}\right)\left(y_{q-t}\right)=a+c y_{q-t} .
$$

Set $g=f-\left(b_{t}+c\right) i_{q}$. We claim that $g$ maps the elements $x_{q+1}, z_{q+1}$ and $y_{j}$, for $1 \leq j \leq q-(t+1)$, inside $R_{q-(t+1)}$. Indeed, if $u$ is in the ideal of $R_{q-(t+1)}$ generated by $x_{q+1}, z_{q+1}$ and $y_{j}$, for $1 \leq j \leq q-(t+1)$, we have

$$
y_{q-t} g(u)=u g\left(y_{q-t}\right) \in R_{q-(t+1)} .
$$

Since by the inductive hypothesis of Theorem 2.3 we have normality of $R_{q-(t+1)}$ and the fact that $R_{q-t}$ is an unprojection of $R_{q-(t+1)}$, using [PR], Remark 1.3.4 (cf. [P1], Lemma 2.1.7) we get that $g(u) \in R_{q-(t+1)}$, which finishes the proof of Proposition 2.12

Corollary 2.13. Fix $s \in \operatorname{Hom}_{R_{0}}\left(L_{q}, R_{0}\right)$ such that $s$ together with $i_{1, q}$ generate the $R_{0}$-module $\operatorname{Hom}_{R_{0}}\left(L_{q}, R_{0}\right)$. Then $\phi_{q}(s)$ together with $i_{q}$ generate the $R_{q}$-module $\operatorname{Hom}_{R_{q}}\left(J_{q}, R_{q}\right)$.

Proof. Assume $f \in \operatorname{Hom}_{R_{q}}\left(J_{q}, R_{q}\right)$. Using Proposition 2.12, there exists $b \in R_{q}$ such that, if we set $g=f-b i_{q}$, we have $g\left(x_{q+1}\right) \in R_{0}$ and $g\left(z_{q+1}\right) \in R_{0}$. Therefore, there exists $g_{1} \in \operatorname{Hom}_{R_{0}}\left(L_{q}, R_{0}\right)$, with $g_{1}\left(x_{q+1}\right)=g\left(x_{q+1}\right)$ and $g_{1}\left(z_{q+1}\right)=g\left(z_{q+1}\right)$. By the assumptions there exist $c_{1}, c_{2} \in R_{0}$ with $g_{1}=c_{1} s+c_{2} i_{1, q}$. As a consequence,

$$
f\left(x_{q+1}\right)=c_{1}\left[\phi_{q}(s)\left(x_{q+1}\right)\right]+\left(b+c_{1}\right) i_{q}\left(x_{q+1}\right) .
$$


Since by the inductive hypothesis $R_{q}$ is a domain, $x_{q+1}$ is a regular element of $R_{q}$. Arguing as in the beginning of the proof of Lemma 2.11 we get

$$
f=c_{1} \phi_{q}(s)+\left(b+c_{1}\right) i_{q},
$$

and the result follows.

Proposition 2.14. The ring $R_{q+1}$ is isomorphic to the unprojection ring of the pair $J_{q} \subset R_{q}$.

Proof. To simplify the notation of the proof we set, for $a, b \in\{x, z\}, Q^{a b}=Q_{i, q+1}^{a b}$. Using [P2], Section $4, \operatorname{Hom}_{R_{0}}\left(L_{q}, R_{0}\right)$ is generated as an $R_{0}$-module by the inclusion map $i_{1, q}$ together with the homomorphism $t: L_{q} \rightarrow R_{0}$ such that

$$
t\left(x_{q+1}\right)=-\left(x_{i} Q^{x z}+z_{i} Q^{z z}\right), \quad t\left(z_{q+1}\right)=x_{i} Q^{x x}+z_{i} Q^{z x}
$$

for all $1 \leq i \leq q$. Notice that these equations correspond exactly to $l_{q+1}$ and $m_{q+1}$. Using Corollary 2.13 $i_{q}$ together with $\phi_{q}(t)$ generate the $R_{q}$-module $\operatorname{Hom}_{R_{q}}\left(J_{q}, R_{q}\right)$, so $\phi_{q}(t)$ can be used to define the unprojection ring.

Fix $1 \leq i \leq q$. We have inside $R_{q}$

$$
x_{q+1}\left[\phi_{q}(t)\left(y_{i}\right)\right]=y_{i}\left[\phi_{q}(t)\left(x_{q+1}\right)\right]=-y_{i}\left(x_{i} Q^{x z}+z_{i} Q^{z z}\right) .
$$

Using the relations $l_{i}=0$ and $m_{i}=0$ which hold in $R_{q}$ (since $1 \leq i \leq q$ ), we get

$$
\begin{aligned}
& -y_{i}\left(x_{i} Q^{x z}+z_{i} Q^{z z}\right)=-Q^{x z}\left(x_{i} y_{i}\right)-Q^{z z}\left(z_{i} y_{i}\right) \\
& =Q^{x z}\left(x_{q+1} Q^{z x}+z_{q+1} Q^{z z}\right)-Q^{z z}\left(x_{q+1} Q^{x x}+z_{q+1} Q^{x z}\right) \\
& =x_{q+1}\left(Q^{x z} Q^{z x}-Q^{x x} Q^{z z}\right) .
\end{aligned}
$$

Hence, since $R_{q}$ is a domain,

$$
\phi_{q}(t)\left(y_{i}\right)-\left(Q^{x z} Q^{z x}-Q^{x x} Q^{z z}\right)=0,
$$

which corresponds exactly to $e_{i q}$. As a consequence, Proposition 2.14 follows.

Proposition 2.15. The ring $R_{q+1}$ is a Gorenstein integral domain, of dimension equal to $\operatorname{dim} R_{0}$, containing $R_{q}$ in a natural way and contained in the field of fractions $K\left(R_{0}\right)$.

Proof. Using Proposition 2.14, we get by the definitions of unprojection ([PR], Section 1 ) that $R_{q}$ is contained in a natural way in $R_{q+1}$ and that $R_{q+1}$ has the same dimension as $R_{q}$; hence by the inductive hypothesis it has the same dimension as $R_{0}$. Moreover, by [PR], Theorem 1.5 and the inductive hypotheses for $R_{q}$ we get that $R_{q+1}$ is Gorenstein, and by $[\mathrm{PR}$, Remark 1.5 that it is also a domain contained in a natural way in the field of fractions $K\left(R_{q}\right)$ of $R_{q}$. Since by the inductive hypothesis $K\left(R_{q}\right)=K\left(R_{0}\right)$, Proposition 2.15 follows.

Proposition 2.16. The sequence $x_{q+1}, z_{q+1}$ is a regular sequence for $R_{q+1}$.

Proof. By Proposition [2.15, $R_{q+1}$ is a Gorenstein integral domain, of dimension equal to $\operatorname{dim} R_{0}$. As a consequence, the result follows by using Proposition 2.8.

Proposition 2.17. There exists a Zariski closed subset $F_{q+1} \subset \operatorname{Spec} R_{q+1}$, with the codimension of $F_{q+1}$ in Spec $R_{q+1}$ at least 2, such that the open subscheme Spec $R_{q+1} \backslash F_{q+1}$ is naturally isomorphic with an open subscheme of $\operatorname{Spec} R_{0}$. 
Proof. By the construction of unprojection,

$$
\operatorname{Spec} R_{q+1} \backslash V\left(x_{q+1}, z_{q+1}, y_{1}, \ldots, y_{q}\right)
$$

is naturally isomorphic to an open subset of $\operatorname{Spec} R_{q}$. Using the inductive hypothesis and Proposition 2.16 the result follows.

Proposition 2.18. The ring $R_{q+1}$ is a normal domain.

Proof. By Proposition 2.15, $R_{q+1}$ is a Gorenstein integral domain. The result follows by combining the normality of $R_{0}$ (Lemma 2.10), Proposition 2.17 and Serre's normality criterion ([BH] , Theorem 2.2 .22$)$.

Proposition 2.19. If $1 \leq i<j \leq n$, then $x_{i}, x_{j}$ is a regular sequence of $R_{q+1}$.

Proof. If this was not true, using the fact that $R_{q+1}$ is Gorenstein (Proposition 2.15) we would have that $V\left(x_{i}, x_{j}\right) \subset$ Spec $R_{q+1}$ would have codimension at most 1 in Spec $R_{q+1}$. Using Proposition 2.17, we get that $x_{i}, x_{j}$ is not a regular sequence for $R_{0}$, contradicting Lemma 2.9.

We have now finished the proof of the inductive step, hence the proof of Theorem 2.3 .

2.2. Generic perfection of $R_{p}$. We fix $n \geq 2$ and $0 \leq p \leq n$. We will prove that the $A_{p}$-module $R_{p}$ is a generically perfect $A_{p}$-module. Recall (BV], Section 3.A) that this means that $R_{p}$ is a perfect $A_{p}$-module and also faithfully flat as a $\mathbb{Z}$ module.

A useful consequence of the generic perfection of $R_{p}$ is that whenever we substitute the variables of the ideal $I_{p}$ with elements of an arbitrary Noetherian ring, we get, under mild conditions, good induced properties of the resulting ideal (cf. $\left[\mathrm{BV}\right.$, Section 3 for precise statements). We will use the generic perfection of $R_{p}$ in Corollary 2.22, Remark 2.23 and Proposition 3.5.

Remark 2.20. Recall ([BV], Section 16.B) that if $A$ is a Noetherian ring and $M$ a finitely generated $A$-module, the grade of $M$ is defined to be the maximal length of an $A$-regular sequence contained in the annihilator ideal Ann $M$ of $M$. If in addition $A$ is a graded Cohen-Macaulay and $M$ is a graded module, we have that the grade of $M$ is equal to $\operatorname{dim} A-\operatorname{dim} A / \mathrm{Ann} M$ (cf. $[\mathrm{BH}$, Corollary 2.1.4). As a consequence, using Theorem 2.3. $R_{p}$ has the grade as an $A_{p}$-module equal to $p+1$.

Proposition 2.21. The $A_{p}$-module $R_{p}$ is generically perfect of the grade $p+1$.

Proof. Using [BV], Proposition 3.2 it is enough to prove that $R_{p}$ is a perfect $A_{p^{-}}$ module, and for every prime integer $q$ the $A_{p} \otimes_{\mathbb{Z}} \mathbb{Z} / q$-module $R_{p} \otimes_{\mathbb{Z}} \mathbb{Z} / q$ is perfect.

Using the remark just before [BV], Proposition 16.20, the perfection of $R_{p}$ as an $A_{p}$-module follows from the Gorensteinness of $R_{p}$ (Theorem 2.3).

Fix an integer prime $q$. It is clear that all the arguments we used to prove Theorem 2.3 also work if we replace $\mathbb{Z}$ by $\mathbb{Z} / q$. As a consequence, we can argue as in the case of $R_{p}$ to get that the $A_{p} \otimes_{\mathbb{Z}} \mathbb{Z} / q$-module $R_{p} \otimes_{\mathbb{Z}} \mathbb{Z} / q$ is perfect, which finishes the proof of the proposition.

Corollary 2.22. Let $k$ be an arbitrary field. The $A_{p} \otimes k$-module $R_{p} \otimes_{\mathbb{Z}} k$ is perfect, of the grade equal to $p+1$. Moreover the $k$-algebra $R_{p} \otimes_{\mathbb{Z}} k$ is Gorenstein. 
Proof. It follows immediately by combining the Gorensteinness of $R_{p}$ (Theorem 2.3) and the generic perfection of $R_{p}$ (Proposition [2.21) with [BV], Theorems 3.3 and 3.6 .

Remark 2.23. Using the construction of unprojection in [KM] which is based on resolution complexes, together with the fact that $J_{p}$ has Koszul complex as its minimal resolution over $A_{p}$ (since it is generated by a regular sequence), we can inductively build a free graded resolution of $R_{p}$ over $A_{p}$. Using [BV], Theorem 3.3 this will give us a free graded resolution of $R_{p} \otimes_{\mathbb{Z}} k$ over $A_{p} \otimes_{\mathbb{Z}} k$, where $k$ is an arbitrary field. We will use this remark in Proposition 3.5 .

More precisely, assume $S$ is a polynomial ring over the integers or over a field, and $I \subset J \subset S$ are two homogeneous ideals of $S$ such that both quotient rings $S / I$ and $S / J$ are Gorenstein and $\operatorname{dim} S / I=\operatorname{dim} S / J+1$. We define $k_{1}, k_{2} \in \mathbb{Z}$ such that $\omega_{S / I}=S / I\left(k_{1}\right)$ and $\omega_{S / J}=S / J\left(k_{2}\right)$ (cf. [BH], p. 140) and assume that $k_{1}>k_{2}$. Moreover, let

$$
0 \rightarrow A_{g} \rightarrow A_{g-1} \rightarrow \cdots \rightarrow A_{1} \rightarrow A_{0} \rightarrow S / J \rightarrow 0
$$

and

$$
0 \rightarrow B_{g-1} \rightarrow \cdots \rightarrow B_{1} \rightarrow B_{0} \rightarrow S / I \rightarrow 0
$$

be the minimal free graded resolutions of $S / J$ and $S / I$ respectively as $S$-modules. Denote by $\widetilde{I} \subset S[v]$ the ideal of the unprojection of the pair $J / I \subset S / I$, where $v$ is a new variable of degree $k_{1}-k_{2}$. Then, whenever $g \geq 3$ (the case $g=2$ is straightforward), a free graded resolution of the quotient ring $S[v] / \widetilde{I}$ as an $S[v]$ module is equal to

$$
0 \rightarrow F_{g} \rightarrow F_{g-1} \rightarrow \cdots \rightarrow F_{1} \rightarrow F_{0} \rightarrow S[v] / \widetilde{I} \rightarrow 0,
$$

where

$$
\begin{aligned}
& F_{0}=B_{0}^{\prime}, \quad F_{1}=B_{1}^{\prime} \oplus A_{1}^{\prime}\left(k_{2}-k_{1}\right), \\
& F_{i}=B_{i}^{\prime} \oplus A_{i}^{\prime}\left(k_{2}-k_{1}\right) \oplus B_{i-1}^{\prime}\left(k_{2}-k_{1}\right) \quad \text { for } 2 \leq i \leq g-2, \\
& F_{g-1}=A_{g-1}^{\prime}\left(k_{2}-k_{1}\right) \oplus B_{g-2}^{\prime}\left(k_{2}-k_{1}\right), \quad F_{g}=B_{g-1}^{\prime}\left(k_{2}-k_{1}\right) ;
\end{aligned}
$$

cf. [KM], p. 307, Equation (3). In the above expression, for an $S$-module $M$ we denoted by $M^{\prime}$ the $S[v]$-module $M \otimes_{S} S[v]$.

For the following we fix an arbitrary field $k$.

Lemma 2.24. The length $n+2^{n}-1$ sequence $z_{1}, \ldots, z_{n}, r_{d_{1} \cdots d_{n}}$ with indices $\left(d_{1}, \ldots, d_{n}\right) \in\{0,1\}^{n}$ and $\left(d_{1}, \ldots, d_{n}\right) \neq(0,0, \ldots, 0)$ is regular for $R_{p} \otimes_{\mathbb{Z}} k$ with respect to any ordering of $i t$.

Proof. Denote by $T \subset R_{p} \otimes_{\mathbb{Z}} k$ the ideal of $R_{p} \otimes_{\mathbb{Z}} k$ generated by the sequence. Since by Corollary $2.22 R_{p} \otimes_{\mathbb{Z}} k$ is Gorenstein, hence Cohen-Macaulay, it is enough to prove that

$$
\operatorname{dim}\left(R_{p} \otimes_{\mathbb{Z}} k\right) / T=\operatorname{dim}\left(R_{p} \otimes_{\mathbb{Z}} k\right)-\left(n+2^{n}-1\right) .
$$

Denote by $T_{1}$ the monomial ideal of $k\left[x_{1}, \ldots, x_{n}, y_{1}, \ldots, y_{p}, r_{00 \cdots 0}\right]$ generated by $x_{i} y_{i}$ for $1 \leq i \leq p, y_{i} y_{j}$ for $1 \leq i<j \leq p$, and $w_{t}$ for $1 \leq t \leq p$, with

$$
w_{t}=r_{00 \cdots 0}\left[\prod_{i=1}^{t-1} x_{i}\right]\left[\prod_{i=t+1}^{n} x_{i}\right] \text {. }
$$


Arguing as in the proof of Proposition 2.8 we get that

$$
\left(R_{p} \otimes_{\mathbb{Z}} k\right) / T \cong k\left[x_{1}, \ldots, x_{n}, y_{1}, \ldots, y_{p}, r_{00 \cdots 0}\right] / T_{1}
$$

and, moreover, that the right hand side ring has the right dimension.

Proposition 2.25. Denote by $W_{1}$ the $k$-vector subspace of $A_{p} \otimes_{\mathbb{Z}} k$ spanned by all $r_{d_{1} \cdots d_{n}}$ with $\left(d_{1}, \ldots, d_{n}\right) \in\{0,1\}^{n}$ and $\left(d_{1}, \ldots, d_{n}\right) \neq(0,0, \ldots 0)$. Assume $1 \leq$ $t \leq 2^{n}-1$ and that $s_{1}, \ldots s_{t}$ are elements of $W_{1}$ which are $k$-linearly independent. Then $s_{1}, \ldots s_{t}$ is a regular sequence for $R_{p} \otimes_{\mathbb{Z}} k$. As a consequence, Corollary 2.22 implies that the $\left(A_{p} \otimes_{\mathbb{Z}} k\right) /\left(s_{1}, \ldots, s_{t}\right)$-module $\left(R_{p} \otimes_{\mathbb{Z}} k\right) /\left(s_{1}, \ldots, s_{t}\right)$ is perfect, of grade equal to $p+1$, and the k-algebra $\left(R_{p} \otimes_{\mathbb{Z}} k\right) /\left(s_{1}, \ldots, s_{t}\right)$ is Gorenstein.

Proof. Since by Corollary $2.22 R_{p} \otimes_{\mathbb{Z}} k$ is Gorenstein, hence Cohen-Macaulay, it is enough to prove that the dimension drops by $t$ when we divide $R_{p} \otimes_{\mathbb{Z}} k$ by the ideal generated by $s_{1}, \ldots, s_{t}$. This follows from Lemma 2.24 after completing $s_{i}$ to a basis of $W_{1}$ and dividing by the ideal generated by the basis together with $z_{1}, \ldots, z_{n}$.

\section{The numerical Campedelli surface Construction}

In this section we work over the field $k=\mathbb{C}$ of complex numbers. We will use the algebra developed in Section 2 in order to prove the existence of numerical Campedelli surfaces with torsion group equal to $\mathbb{Z} / 6$.

We define the polynomial ring

$$
A_{4}^{s}=k\left[x_{1}, \ldots, x_{4}, z_{1} \ldots, z_{4}, y_{1}, \ldots, y_{4}\right]
$$

( $s$ for specific), and we assign degree 1 to each variable $x_{i}$ and $z_{i}$, for $1 \leq i \leq 4$, and degree 2 to each variable $y_{i}$, for $1 \leq i \leq 4$.

Remark 3.1. This is a different choice of degrees for the variables from the one made in Section 2, In Section 2, it was useful, for technical reasons, to give the variables $r_{d_{1} \ldots d_{n}}$ positive degrees. However, for the Campedelli cover construction they have to be constants.

Let $G$ be the cyclic group of order 6 and denote by $g$ a generator of $G$. We define a linear action of $G$ on $A_{4}^{s}$ by

$$
\begin{array}{llrl}
\left(g x_{1}, g x_{2}, g x_{3}\right) & =\left(-x_{2},-x_{3},-x_{1}\right), & & g x_{4}=-x_{4}, \\
\left(g z_{1}, g z_{2}, g z_{3}\right) & =\left(z_{2}, z_{3}, z_{1}\right), & & g z_{4}=z_{4}, \\
\left(g y_{1}, g y_{2}, g y_{3}\right)=\left(-y_{2},-y_{3},-y_{1}\right), & & g y_{4}=-y_{4} .
\end{array}
$$

Consider the 16-dimensional $k$-vector subspace of $A_{4}^{s}$ spanned by the monomials $a_{1} a_{2} a_{3} a_{4}$, where $a_{i} \in\left\{x_{i}, z_{i}\right\}$. It is easy to see that it is $G$-invariant and that its $k$-vector subspace consisting of the $G$-invariant polynomials has $F_{1}, \ldots, F_{4}$ as a $k$-basis, where

$$
\begin{aligned}
& F_{1}=x_{1} x_{2} x_{3} x_{4}, \quad F_{2}=\left(x_{1} x_{2} z_{3}+x_{1} z_{2} x_{3}+z_{1} x_{2} x_{3}\right) z_{4}, \\
& F_{3}=\left(x_{1} z_{2} z_{3}+z_{1} x_{2} z_{3}+z_{1} z_{2} x_{3}\right) x_{4}, \quad F_{4}=z_{1} z_{2} z_{3} z_{4} .
\end{aligned}
$$

Fix $\left(r_{1}, \ldots, r_{4}\right) \in k^{4}$ nonzero. We set

$$
Q^{s}=Q^{s}\left(r_{t}\right)=\sum_{i=1}^{4} r_{i} F_{i} \in A_{4}^{s} .
$$


The polynomial $Q^{s}$ is homogeneous of degree 4. Similar to item (5) of Notation 2.1. for each $1 \leq i<j \leq n$, let

$$
Q_{i j}^{s, x x}=\frac{\partial^{2} Q^{s}}{\partial x_{i} \partial x_{j}}, \quad Q_{i j}^{s, x z}=\frac{\partial^{2} Q^{s}}{\partial x_{i} \partial z_{j}}, \quad Q_{i j}^{s, z x}=\frac{\partial^{2} Q^{s}}{\partial z_{i} \partial x_{j}} \quad \text { and } \quad Q_{i j}^{s, z z}=\frac{\partial^{2} Q^{s}}{\partial z_{i} \partial z_{j}} .
$$

We clearly have

$$
Q^{s}=x_{i} x_{j} Q_{i j}^{s, x x}+x_{i} z_{j} Q_{i j}^{s, x z}+z_{i} x_{j} Q_{i j}^{s, z x}+z_{i} z_{j} Q_{i j}^{s, z z} .
$$

Consider, for $1 \leq i<j \leq 4$, the $5 \times 5$ skew-symmetric matrix

$$
M_{i j}^{s}=\left(\begin{array}{ccccc}
0 & x_{i} & z_{i} & -x_{j} & -z_{j} \\
& 0 & y_{j} & Q_{i j}^{s, z z} & -Q_{i j}^{s, z x} \\
& & 0 & -Q_{i j}^{s, x z} & Q_{i j}^{s, x x} \\
& -\mathrm{sym} & & 0 & -y_{i} \\
& & & & 0
\end{array}\right)
$$

with entries in $A_{4}^{s}$. We denote by $I_{4}^{s}$ the ideal of $A_{4}^{s}$ generated by all the submaximal Pfaffians of $M_{i j}^{s}$ for all values $1 \leq i<j \leq 4$.

The analogue of item (6) of Notation 2.1 is true, and we denote, for $1 \leq i<j \leq 4$, by $e_{i j}^{s}$ the Pfaffian of $M_{i j}^{s}$ involving $y_{i} y_{j}$ with coefficient 1 , by $l_{i}^{s}$ the Pfaffian of $M_{1 i}^{s}$ (or of $M_{12}^{s}$ if $i=1$ ) involving $x_{i} y_{i}$ with coefficient 1 , and by $m_{i}^{s}$ the Pfaffian of $M_{1 i}^{s}$ (or of $M_{12}^{s}$ if $i=1$ ) involving $z_{i} y_{i}$ with coefficient 1. See (3.9) below for the explicit formulas of $l_{i}^{s}, m_{i}^{s}$ and $e_{i j}^{s}$.

It is also clear that

$$
I_{4}^{s}=\left(e_{i j}^{s}, l_{t}^{s}, m_{t}^{s}\right) \subset A_{4}^{s}
$$

with indices $1 \leq i<j \leq 4$ and $1 \leq t \leq 4$.

We denote $R_{4}^{s}=A_{4}^{s} / I_{4}^{s}$ as the quotient ring (which, of course, also depends on the choice of parameter values $\left.\left(r_{t}\right)\right)$.

Proposition 3.2. a) For any $\left(r_{1}, \ldots, r_{4}\right) \in k^{4}$, $\operatorname{dim} R_{4}^{s} \geq 7$.

b) Whenever $\operatorname{dim} R_{4}^{s}=7, R_{4}^{s}$ is a Gorenstein ring and a perfect $A_{4}^{s}$-module.

c) There exist parameter values $\left(r_{t}\right)$ such that $\operatorname{dim} R_{4}^{s}=7$.

d) For general parameter values $\left(r_{1}, \ldots, r_{4}\right) \in k^{4}$ (in the sense of being outside a proper Zariski closed subset of $k^{4}$ ) the ring $R_{4}^{s}$ is Gorenstein with $\operatorname{dim} R_{4}^{s}=7$.

Proof. Since the polynomial $Q^{s}$ defined in (3.1) can be obtained as a specialization of the polynomial $Q$ defined in (4) of Notation 2.1, it is clear that there exist $s_{1}, \ldots, s_{t} \in W_{1}, k$-linearly independent (where $W_{1}$ was defined in Proposition 2.25), such that for $\left(r_{1}, \ldots, r_{4}\right) \in k^{4}$, Spec $R_{4}^{s}$ is isomorphic to the fiber over the point with coordinates $r_{d_{1} \ldots d_{4}}$ corresponding to $\left(r_{1}, \ldots, r_{4}\right)$ of the natural map

$$
\operatorname{Spec}\left(R_{4} \otimes_{\mathbb{Z}} k\right) /\left(s_{1}, \ldots, s_{t}\right) \rightarrow \operatorname{Spec} k\left[r_{d_{1} \ldots d_{4}}\right] /\left(s_{1}, \ldots, s_{t}\right)
$$

induced by the inclusion

$$
k\left[r_{d_{1} \ldots d_{4}}\right] /\left(s_{1}, \ldots, s_{t}\right) \rightarrow\left(R_{4} \otimes_{\mathbb{Z}} k\right) /\left(s_{1}, \ldots, s_{t}\right) .
$$

By $[\mathrm{L}$, Theorem 4.3.12, for a morphism $f: X \rightarrow Y$ between locally Noetherian schemes and a point $x \in X$ we have

$$
\operatorname{dim} \mathcal{O}_{X_{y}, x} \geq \operatorname{dim} \mathcal{O}_{X, x}-\operatorname{dim} \mathcal{O}_{Y, y},
$$

where $y=f(x)$ and $X_{y}$ is the fiber of $f$ over $y$. Combining it with Proposition 2.25 we get part a).

Part b) follows similarly by combining Proposition 2.25] and [BV], Theorem 3.5. 
For part c) we make a specific choice of parameters $r_{1}=1$ and $r_{i}=0$ for $2 \leq i \leq 4$. We will prove that $\operatorname{dim} R_{4}^{s}=7$. For that, it is enough to prove that $\operatorname{dim} R_{4}^{s} /\left(z_{1}, z_{2}, z_{3}, z_{4}\right)=3$. Arguing as in the proof of Proposition 2.8. it is easy to see (compare also (3.9)) that

$$
R_{4}^{s} /\left(z_{1}, z_{2}, z_{3}, z_{4}\right) \cong k\left[x_{1}, \ldots, x_{4}, y_{1}, \ldots, y_{4}\right] / T,
$$

where $T$ is the monomial ideal of $k\left[x_{1}, \ldots, x_{4}, y_{1}, \ldots, y_{4}\right]$ generated by the elements $x_{i} y_{i}$, for $1 \leq i \leq 4$, together with $y_{i} y_{j}$, for $1 \leq i<j \leq 4$, and together with $w_{t}$, for $1 \leq t \leq 4$, where

$$
w_{t}=\left[\prod_{i=1}^{t-1} x_{i}\right]\left[\prod_{i=t+1}^{4} x_{i}\right]
$$

and that we indeed have the right dimension.

Using semicontinuity of the fiber dimension (cf. Ei], Corollary 14.9) and parts a), b), and c), we have that part d) follows, which completes the proof of Proposition 3.2 .

Remark 3.3. A different way of arguing for the proof of Proposition 3.2 is to suitably modify the arguments used in the proof of Theorem [2.3 One should be able to obtain in this manner the more precise result that $R_{4}^{s}$ is Gorenstein with $\operatorname{dim} R_{4}^{s}=7$ whenever there exists $i, 1 \leq i \leq 4$, with $r_{i}$ nonzero. We will not use that in the following.

Denote by $\zeta \in k$ a fixed primitive 6 th root of unity. We consider the following homogeneous elements $n_{i j} \in A_{4}^{s}$ of degree 1 :

$$
\begin{aligned}
& n_{01}=z_{1}+z_{2}+z_{3}, \quad n_{02}=z_{4}, \quad n_{11}=x_{1}+\zeta^{2} x_{2}+\zeta^{4} x_{3}, \\
& n_{21}=z_{1}+\zeta^{4} z_{2}+\zeta^{2} z_{3}, \quad n_{31}=x_{1}+x_{2}+x_{3}, \quad n_{32}=x_{4}, \\
& n_{41}=z_{1}+\zeta^{2} z_{2}+\zeta^{4} z_{3}, \quad n_{51}=x_{1}+\zeta^{4} x_{2}+\zeta^{2} x_{3} .
\end{aligned}
$$

By construction, each $n_{i j}$ is an eigenvector for the action of $g \in G$ with eigenvalue equal to $\zeta^{i}$; that is,

$$
g n_{i j}=\zeta^{i} n_{i j}
$$

We fix 4 more complex numbers $\left(r_{5}, \ldots, r_{8}\right) \in k^{4}$, and we define 4 homogeneous elements $h_{i}=h_{i}\left(r_{5}, \ldots, r_{8}\right) \in A_{4}^{s}$, for $1 \leq i \leq 4$, by

$$
\begin{aligned}
& h_{1}=n_{01}, \quad h_{2}=n_{02}, \quad h_{3}=n_{32}+r_{5} n_{31}, \\
& h_{4}=y_{4}+r_{6}\left(y_{1}+y_{2}+y_{3}\right)+r_{7} n_{11} n_{21}+r_{8} n_{41} n_{51} .
\end{aligned}
$$

We have that, for $1 \leq i \leq 4$, the element $h_{i}$ is an eigenvector for $g$ with eigenvalue equal to 1 for $h_{1}$ and $h_{2}$ and eigenvalue equal to -1 for $h_{3}$ and $h_{4}$.

We denote by $T^{s} \subset A_{4}^{s}$ the homogeneous ideal

$$
T^{s}=T^{s}\left(r_{t}\right)=I_{4}^{s}+\left(h_{1}, \ldots, h_{4}\right) \subset A_{4}^{s} .
$$

Moreover, we denote by $A$ the polynomial subring

$$
A=k\left[x_{1}, x_{2}, x_{3}, z_{1}, z_{2}, y_{1}, y_{2}, y_{3}\right] \subset A_{4}^{s}
$$

with the weighting of the variables induced by that of $A_{4}^{s}$. For fixed general parameter values $\left(r_{1}, \ldots, r_{8}\right) \in k^{8}$, the composition

$$
A \rightarrow A_{4}^{s} \rightarrow A_{4}^{s} / T^{s}
$$


is surjective (where the first map is the natural inclusion and the second is the natural projection), so we get an induced isomorphism

$$
\frac{A}{L} \cong \frac{A_{4}^{s}}{T^{s}}
$$

where $L \subset A$ is the kernel of the composition.

Proposition 3.4. a) For any choice of parameter values $\left(r_{1}, \ldots, r_{8}\right) \in k^{8}$ we have $\operatorname{dim} A_{4}^{s} / T^{s} \geq 3$, and whenever $\operatorname{dim} A_{4}^{s} / T^{s}=3$ we have that $A_{4}^{s} / T^{s}$ is a Gorenstein ring, perfect as $A_{4}^{s}$-module.

b) There exist parameter values $\left(r_{t}\right)$ such that $\operatorname{dim} A_{4}^{s} / T^{s}=3$.

c) For general parameter values $\left(r_{1}, \ldots, r_{8}\right) \in k^{8}$ (in the sense of being outside a proper Zariski closed subset of $\left.k^{8}\right), \operatorname{dim} A_{4}^{s} / T^{s}=3$ and $A_{4}^{s} / T^{s}$ is a Gorenstein ring.

Proof. Part a) follows immediately from Proposition 3.2 by noticing that $A_{4}^{s} / T^{s}$ is isomorphic to $R_{4}^{s} /\left(h_{1}, \ldots, h_{4}\right)$.

For part b) we fix the parameter values $r_{1}=r_{4}=1$ and $r_{j}=0$, for $2 \leq j \leq 8$ with $j \neq 4$. By (3.4) $A_{4}^{s} / T^{s} \cong A / L$, where $L$ is the ideal of $A$ generated by

$$
\begin{gathered}
\left\{x_{1} y_{1}, z_{1} y_{1}, x_{2} y_{2}, z_{2} y_{2}, x_{3} y_{3}, z_{1} y_{3}+z_{2} y_{3}, z_{1}^{2} z_{2}+z_{1} z_{2}^{2}, x_{1} x_{2} x_{3}, y_{1} y_{2}\right. \\
\left.y_{1} y_{3}, x_{2} x_{3} z_{1} z_{2}+x_{2} x_{3} z_{2}^{2}, y_{2} y_{3}, x_{1} x_{3} z_{1}^{2}+x_{1} x_{3} z_{1} z_{2}, x_{1} x_{2} z_{1} z_{2}\right\} .
\end{gathered}
$$

It is easily checked that each minimal associated prime of $L$ has codimension 5 in $A$; hence

$$
\operatorname{dim} A / L=3 .
$$

Part c) is an immediate consequence of parts a) and b), arguing as in the proof of Proposition 3.2

Proposition 3.5. For general parameter values $\left(r_{1}, \ldots, r_{8}\right) \in k^{8}$ the minimal graded resolution of $A / L$ as an $A$-module is equal to

$$
\begin{aligned}
& 0 \rightarrow A(-12) \rightarrow A(-9)^{8} \oplus A(-8)^{6} \rightarrow A(-8)^{3} \oplus A(-7)^{24} \oplus A(-6)^{8} \\
& \rightarrow A(-6)^{8} \oplus A(-5)^{24} \oplus A(-4)^{3} \rightarrow A(-4)^{6} \oplus A(-3)^{8} \rightarrow A .
\end{aligned}
$$

Moreover, the dualising module of $A / L$ is equal to $(A / L)(1)$ and the Hilbert series of $A / L$ as a graded $A$-module is equal to

$$
\frac{t^{4}+2 t^{3}+6 t^{2}+2 t+1}{(1-t)^{3}} \in \mathbb{Q}(t) .
$$

Proof. Using Remark 2.23, we can easily calculate inductively the minimal graded resolution of the generically perfect (Proposition 2.21) module $R_{4}$ over $A_{4}$. Equation (3.5) follows by combining Proposition 3.4. BV], Theorem 3.5, and the easily observed fact that the minimal graded resolution of $R_{4}$ over $A_{4}$ remains homogeneous and minimal. The other conclusions of Proposition 3.5 follow easily from (3.5).

Definition 3.6. For general $\left(r_{1}, \ldots, r_{8}\right) \in k^{8}$ we denote by $S$ the scheme

$$
S=S\left(r_{t}\right)=\operatorname{Proj} A_{4}^{s} / T^{s} \subset \mathbb{P}\left(1^{8}, 2^{4}\right) .
$$


Our main aim is to prove that $S$ is an irreducible nonsingular surface with invariants $p_{g}=5, q=0, K^{2}=12$ and a trivial algebraic fundamental group, which is an étale 6 to 1 cover of a numerical Campedelli surface.

Remark 3.7. By (3.4), $S$ has an embedding as a nondegenerate subscheme

$$
S \subset \mathbb{P}\left(1^{5}, 2^{3}\right) .
$$

Proposition 3.8. a) The homogeneous coordinate ring of the embedding $S \subset$ $\mathbb{P}\left(1^{8}, 2^{4}\right)$ is isomorphic to $A_{4}^{s} / T^{s}$.

b) The scheme $S$ is a projective purely 2-dimensional scheme over $k$. Moreover, $S$ is connected and $H^{1}\left(S, \mathcal{O}_{S}(t)\right)=0$ for all $t \in \mathbb{Z}$.

c) The dualising sheaf $\omega_{S}$ is isomorphic to $\mathcal{O}_{S}(1)$ as an $\mathcal{O}_{S}$-module.

Proof. The graded ring $A_{4}^{s} / T^{s}$ is Gorenstein (Proposition 3.4), hence saturated. As a consequence, part a) follows.

Using part a) the homogeneous coordinate ring of the embedding $S \subset \mathbb{P}\left(1^{8}, 2^{4}\right)$ is Gorenstein, hence Cohen-Macaulay. It is then well known (cf. Do, Ei], Ch. 18) that the conclusions of part b) follow. It is also well-known that part c) follows immediately from Proposition 3.5 .

In the following we will also need the affine cone $S^{c} \subset \mathbb{A}^{12}$ over $S \subset \mathbb{P}\left(1^{8}, 2^{4}\right)$, so we set

$$
S^{c}=V\left(T^{s}\right) \subset \mathbb{A}^{12} .
$$

We denote by $S_{c l}^{c}$ the set of closed points of $S^{c}$, and by $S_{c l}$ the set of closed points of $S$. Since $k=\mathbb{C}$ is algebraically closed, we can identify $S_{c l}^{c}$ with the set of points

$$
P=\left(a_{1}, \ldots, a_{4} ; \quad b_{1}, \ldots, b_{4} ; c_{1}, \ldots, c_{4}\right) \in k^{12}
$$

such that $f(P)=0$ for every $f \in T^{s}$.

By definition, $S_{c l}$ is the quotient of $S_{c l}^{c} \backslash\{0\}$ under the group action

$$
k^{*} \times\left(S_{c l}^{c} \backslash\{0\}\right) \rightarrow\left(S_{c l}^{c} \backslash\{0\}\right)
$$

with

$$
h P=\left(h a_{1}, \ldots, h a_{4} ; h b_{1}, \ldots, h b_{4} ; h^{2} c_{1}, \ldots, h^{2} c_{4}\right)
$$

for $h \in k^{*}$ and $P \in S_{c l}^{c}$ as in (3.7).

Since by Proposition 3.14 below the ideal $T^{s} \subset R_{4}^{s}$ is $G$-invariant, there is an induced $G$ action $G \times R_{4}^{s} / T^{s} \rightarrow R_{4}^{s} / T^{s}$, which induces in a natural way two group actions: $G \times S_{c l}^{c} \rightarrow S_{c l}^{c}$ and $G \times S_{c l} \rightarrow S_{c l}$.

Explicitly, for $P \in S_{c l}^{c}$ as in (3.7) we have

$$
g P=\left(-a_{3},-a_{1},-a_{2},-a_{4} ; b_{3}, b_{1}, b_{2}, b_{4} ;-c_{3},-c_{2},-c_{1},-c_{4}\right) .
$$

Lemma 3.9. For general values of parameters $\left(r_{1}, \ldots, r_{8}\right) \in k^{8}$ there is no nonzero point $P \in S_{c l}^{c}$ (notation for $P$ as in (3.7)) such that $a_{i}=b_{i}=0$ for all $1 \leq i \leq 4$.

Proof. Indeed, if all $a_{i}=b_{i}=0$ we have by looking at $e_{i j}^{s}$, for $1 \leq i<j \leq 4$ (cf. (3.9)), that at least three of the four $c_{t}$ are 0 , and then by looking at the polynomial $h_{4}$ we get that the remaining $c_{t}$ is also 0 , a contradiction to $P \neq 0$. 
Proposition 3.10. Consider $S \subset \mathbb{P}\left(1^{5}, 2^{3}\right)$ as in (3.6). Denote by $S_{1}^{c} \subset \mathbb{A}^{8}$ the affine cone over $S$. The scheme $S_{1}^{c}$ is smooth outside the vertex of the cone.

Proof. Unfortunately, we were only able to prove Proposition 3.10 with the help of the computer algebra program Singular GPS01. We took a similar approach as in R2, p. 18 and worked over the finite field of $\mathbb{Z} / 103$ after putting values for parameters $r_{7}=r_{8}=0$, in order to have everything defined over $\mathbb{Z}$.

Remark 3.11. Using the birational character of unprojection it is not hard to specify inductively (for general values of the parameters $\left(r_{t}\right)$ ) the singularities of the affine cone over the 6 -fold $V\left(I_{t}^{s}\right)$ for $t=0, \ldots, 4$, where $I_{t}^{s}$ are the precise analogues of the ideals $I_{t}$ defined in Section 2. With a little more effort, one can also specify inductively the singularities of the cone over the 3 -fold $V\left(I_{t}^{s}+\left(h_{1}, h_{2}, h_{3}\right)\right)$. Since $h_{2}=z_{4}$ vanishes, the trick here is to start from the codimension 2 ideal $\left(l_{4}^{s}, m_{4}^{s}\right)$ and then inductively unproject $V\left(x_{1}, z_{1}, y_{4}\right), V\left(x_{2}, z_{2}, y_{4}, y_{1}\right)$ and finally $V\left(x_{3}, z_{1}+z_{2}, y_{4}, y_{1}, y_{2}\right)$. What, unfortunately, we were not able to do was to find a way to deduce the nonsingularity (outside the vertex of the affine cone) of the surface from the singularity calculations of the 3 -fold.

Theorem 3.12. Fix general values of parameters $\left(r_{1}, \ldots, r_{8}\right) \in k^{8} . S=S\left(r_{t}\right)$ is an irreducible minimal nonsingular surface of general type with $p_{g}=5, q=0, K^{2}=12$ and canonical ring isomorphic to $A_{4}^{s} / T^{s}$.

Proof. By combining Lemma 3.9 and Proposition 3.10 we get that the scheme $S$ is smooth. Since $S$ is also connected (Proposition [3.8), it follows that $S$ is an irreducible nonsingular surface.

By Proposition 3.8 the dualising sheaf $\omega_{S}$ is isomorphic to $\mathcal{O}_{S}(1)$. Using Lemma $3.9 \mathcal{O}_{S}(1)$ is globally generated. As a consequence,

$$
\mathcal{O}_{S}(1)^{\otimes n} \cong \mathcal{O}_{S}(n)
$$

for all $n \geq 1$, hence $A_{4}^{s} / T^{s}$ is isomorphic to the canonical ring of $S$. Therefore $\omega_{S}$ is ample, which implies that $S$ is minimal.

Since the irregularity $q$ of $S$ is 0 (because by Proposition $3.8 h^{1}\left(S, \mathcal{O}_{S}\right)=0$ ), the properties $p_{g}=5, K^{2}=12$ follow by comparing the Hilbert series calculation of Proposition 3.5] with [R1], Example 3.5.

Remark 3.13. We will prove below that $S$ has trivial algebraic fundamental group (see the proof of Theorem 3.16).

Our next aim is to prove that $S$ is an étale 6 to 1 cover of a numerical Campedelli surface.

Proposition 3.14. Assume $g_{1} \in G$ and $u \in T^{s}$; then $g_{1} u \in T^{s}$.

Proof. Since $G=\langle g\rangle$, it is enough to check that $g u \in T^{s}$, where $u$ is one of the generators of $I_{4}^{s}$ appearing in (3.2).

It is easy to check (compare (3.9)) that for $i \in\{1,2,3\}$ we have

$$
g l_{i}^{s}=l_{t}^{s}, \quad g m_{i}^{s}=-m_{t}^{s}, \quad g e_{i 4}^{s}=e_{t 4}^{s},
$$

where $t \in\{1,2,3\}$ is uniquely specified by $t \equiv i+1 \bmod 3$, and also that

$$
g l_{4}^{s}=l_{4}^{s}, \quad g m_{4}^{s}=-m_{4}^{s}, \quad g e_{12}^{s}=e_{23}^{s}, \quad g e_{13}^{s}=e_{12}^{s}, \quad g e_{23}^{s}=e_{13}^{s} .
$$

A more conceptual proof can be given by arguing that due to the $G$-invariance of $Q^{s}$, the action of $g$ interchanges (up to sign) the set of $Q_{i j}^{s, a b}$ (for $a, b \in\{x, z\}$ ), 
and we use that to argue that the action of $g$ interchanges (up to sign differences of whole columns or rows) the set of the matrices $M_{i j}^{s}$.

The proof of the following proposition will be given in Subsection 3.1.

Proposition 3.15. Fix general values of the parameters $\left(r_{1}, \ldots, r_{8}\right) \in k^{8}$. If $g_{1} \in$ $G$ is not the identity element and $u \in S_{c l}$, we have $g_{1} u \neq u$. In other words, the action of $G$ on $S_{c l}$ is basepoint free.

The following is our main result about the existence of numerical Campedelli surfaces with algebraic fundamental group equal to $\mathbb{Z} / 6$.

Theorem 3.16. For general $\left(r_{1}, \ldots, r_{8}\right) \in k^{8}$, the action of $G$ on $S$ is basepoint free. As a consequence, the quotient surface $S / G$ is a smooth irreducible minimal complex surface of general type with $p_{g}=q=0$ and $K^{2}=2$ (i.e., a numerical Campedelli surface). Moreover $S / G$ has both algebraic fundamental group and torsion group isomorphic to $\mathbb{Z} / 6$.

Proof. Fix general $\left(r_{1}, \ldots, r_{8}\right) \in k^{8}$. By Proposition 3.15, the action of $G$ on $S$ is basepoint free. Hence using Theorem 3.12 $S / G$ is a smooth irreducible surface. Denote by $\pi: S \rightarrow S / G$ the natural projection map. Since $\pi$ is étale $\pi^{*}\left(\omega_{S / G}\right) \cong \omega_{S}$ (cf. MP, p. 3), and since by the proof of Theorem $3.12 \omega_{S}$ is ample, we have that $\omega_{S / G}$ is ample (cf. [Ha], Exerc. III.5.7). Hence $S / G$ is a minimal surface of general type.

The invariants of $S / G$ follow from those of $S$ calculated in Theorem 3.12, Indeed, $\pi$ surjective and $q(S)=0$ imply $q(S / G)=0$, and $\pi$ étale 6 to 1 implies $K_{S}^{2}=6 K_{S / G}^{2}$ and $\chi(S)=6 \chi(S / G)$.

To prove that the algebraic fundamental group of $S / G$ is equal to $G$, it is enough to show that $\pi_{1}^{\text {alg }} S=0$. Assume it is not. Then the group $\pi_{1}^{\text {alg }}(S / G)$ has $6\left|\pi_{1}^{\text {alg }} S\right| \geq$ 12 elements, which contradicts the fact that a Campedelli surface has algebraic fundamental group consisting of at most 9 elements (cf. BPHV], Chap. VII.10).

Since the torsion group of $S / G$ is the largest abelian quotient of $\pi_{1}^{\text {alg }}(S / G)$ (cf. $[\mathrm{MP}]$, p. 16 ), we get that $S / G$ has a torsion group isomorphic to $\mathbb{Z} / 6$, which finishes the proof of Theorem 3.16

Remark 3.17. It can be shown that the $\left(k^{*}\right)^{5}$ action $A_{4}^{s}$ defined by

$$
g x_{i}=g_{1} x_{i}, \quad g z_{i}=g_{2} z_{i}, \quad g y_{i}=\left(g_{1} g_{2} g_{3}\right) y_{i}
$$

for $1 \leq i \leq 3$, and

$$
g x_{4}=g_{4} x_{4}, \quad g z_{4}=g_{5} z_{4}, \quad g y_{4}=\frac{g_{1}^{2} g_{2}^{2} g_{3}}{g_{4} g_{5}} y_{4},
$$

where $g=\left(g_{1}, \ldots, g_{5}\right) \in\left(k^{*}\right)^{5}$, respects our construction, and the induced action on the space of parameters $r_{1}, \ldots, r_{8}$ has 1-dimensional kernel. As a consequence, the number of moduli of our family is at most 4. As already observed in the Introduction, it follows that there exist numerical Campedelli surfaces with torsion $\mathbb{Z} / 6$ that cannot be obtained by our construction. 
3.1. The proof of Proposition 3.15, For the proof of Proposition 3.15] we will need the following formulas:

$$
\begin{aligned}
& l_{1}^{s}=x_{2} x_{3} z_{4} r_{2}+x_{3} x_{4} z_{2} r_{3}+x_{2} x_{4} z_{3} r_{3}+z_{2} z_{3} z_{4} r_{4}+x_{1} y_{1}, \\
& l_{2}^{s}=x_{1} x_{3} z_{4} r_{2}+x_{3} x_{4} z_{1} r_{3}+x_{1} x_{4} z_{3} r_{3}+z_{1} z_{3} z_{4} r_{4}+x_{2} y_{2}, \\
& l_{3}^{s}=x_{1} x_{2} z_{4} r_{2}+x_{2} x_{4} z_{1} r_{3}+x_{1} x_{4} z_{2} r_{3}+z_{1} z_{2} z_{4} r_{4}+x_{3} y_{3}, \\
& l_{4}^{s}=x_{2} x_{3} z_{1} r_{2}+x_{1} x_{3} z_{2} r_{2}+x_{1} x_{2} z_{3} r_{2}+z_{1} z_{2} z_{3} r_{4}+x_{4} y_{4}, \\
& m_{1}^{s}=-x_{2} x_{3} x_{4} r_{1}-x_{3} z_{2} z_{4} r_{2}-x_{2} z_{3} z_{4} r_{2}-x_{4} z_{2} z_{3} r_{3}+z_{1} y_{1}, \\
& m_{2}^{s}=-x_{1} x_{3} x_{4} r_{1}-x_{3} z_{1} z_{4} r_{2}-x_{1} z_{3} z_{4} r_{2}-x_{4} z_{1} z_{3} r_{3}+z_{2} y_{2}, \\
& m_{3}^{s}=-x_{1} x_{2} x_{4} r_{1}-x_{2} z_{1} z_{4} r_{2}-x_{1} z_{2} z_{4} r_{2}-x_{4} z_{1} z_{2} r_{3}+z_{3} y_{3}, \\
& m_{4}^{s}=-x_{1} x_{2} x_{3} r_{1}-x_{3} z_{1} z_{2} r_{3}-x_{2} z_{1} z_{3} r_{3}-x_{1} z_{2} z_{3} r_{3}+z_{4} y_{4}, \\
& e_{12}^{s}=-x_{3}^{2} z_{4}^{2} r_{2}^{2}+x_{3}^{2} x_{4}^{2} r_{1} r_{3}-x_{3} x_{4} z_{3} z_{4} r_{2} r_{3}-x_{4}^{2} z_{3}^{2} r_{3}^{2} \\
& +x_{3} x_{4} z_{3} z_{4} r_{1} r_{4}+z_{3}^{2} z_{4}^{2} r_{2} r_{4}+y_{1} y_{2} \\
& e_{13}^{s}=-x_{2}^{2} z_{4}^{2} r_{2}^{2}+x_{2}^{2} x_{4}^{2} r_{1} r_{3}-x_{2} x_{4} z_{2} z_{4} r_{2} r_{3}-x_{4}^{2} z_{2}^{2} r_{3}^{2} \\
& +x_{2} x_{4} z_{2} z_{4} r_{1} r_{4}+z_{2}^{2} z_{4}^{2} r_{2} r_{4}+y_{1} y_{3} \\
& e_{14}^{s}=x_{2}^{2} x_{3}^{2} r_{1} r_{2}-x_{3}^{2} z_{2}^{2} r_{2} r_{3}-x_{2} x_{3} z_{2} z_{3} r_{2} r_{3}-x_{2}^{2} z_{3}^{2} r_{2} r_{3} \\
& +x_{2} x_{3} z_{2} z_{3} r_{1} r_{4}+z_{2}^{2} z_{3}^{2} r_{3} r_{4}+y_{1} y_{4}, \\
& e_{23}^{s}=-x_{1}^{2} z_{4}^{2} r_{2}^{2}+x_{1}^{2} x_{4}^{2} r_{1} r_{3}-x_{1} x_{4} z_{1} z_{4} r_{2} r_{3}-x_{4}^{2} z_{1}^{2} r_{3}^{2} \\
& +x_{1} x_{4} z_{1} z_{4} r_{1} r_{4}+z_{1}^{2} z_{4}^{2} r_{2} r_{4}+y_{2} y_{3}, \\
& e_{24}^{s}=x_{1}^{2} x_{3}^{2} r_{1} r_{2}-x_{3}^{2} z_{1}^{2} r_{2} r_{3}-x_{1} x_{3} z_{1} z_{3} r_{2} r_{3}-x_{1}^{2} z_{3}^{2} r_{2} r_{3} \\
& +x_{1} x_{3} z_{1} z_{3} r_{1} r_{4}+z_{1}^{2} z_{3}^{2} r_{3} r_{4}+y_{2} y_{4}, \\
& e_{34}^{s}=x_{1}^{2} x_{2}^{2} r_{1} r_{2}-x_{2}^{2} z_{1}^{2} r_{2} r_{3}-x_{1} x_{2} z_{1} z_{2} r_{2} r_{3}-x_{1}^{2} z_{2}^{2} r_{2} r_{3} \\
& +x_{1} x_{2} z_{1} z_{2} r_{1} r_{4}+z_{1}^{2} z_{2}^{2} r_{3} r_{4}+y_{3} y_{4} .
\end{aligned}
$$

By (3.3) we have $h_{1}=z_{1}+z_{2}+z_{3}, h_{2}=z_{4}$ and $h_{3}=x_{4}+r_{5}\left(x_{1}+x_{2}+x_{3}\right)$.

We set $u_{i} \in k^{12}$, for $1 \leq i \leq 4$, to be the vector with 1 on the coordinate corresponding to $x_{i}$ and 0 elsewhere, $v_{i} \in k^{12}$ to be the vector with 1 on the coordinate corresponding to $z_{i}$ and 0 elsewhere, and $w_{i} \in k^{12}$ to be the vector with 1 on the coordinate corresponding to $y_{i}$ and 0 elsewhere.

Set $V_{1}$ to be the vector space spanned by $u_{1}, u_{2}, u_{3}, V_{2}$ to be the vector space spanned by $v_{1}, v_{2}, v_{3}, V_{3}$ to be the vector space spanned by $u_{4}, V_{4}$ to be the vector space spanned by $v_{4}, V_{5}$ to be the vector space spanned by $w_{1}, w_{2}, w_{3}$ and $V_{6}$ to be the vector space spanned by $w_{4}$. We have that, for $1 \leq i \leq 6$, the vector space $V_{i}$ is $G$-invariant. More precisely, using (3.8) we get

$$
g u_{i}=-u_{t}, \quad g v_{i}=v_{t}, \quad g w_{i}=-w_{t},
$$

where $t \in\{1,2,3\}$ is uniquely specified by $t \equiv i+1 \bmod 3$, and

$$
g u_{4}=-u_{4}, \quad g v_{4}=v_{4}, \quad g w_{4}=-w_{4} .
$$

Since every element of $G$ different from the identity has a power equal to $g^{2}$ or to $g^{3}$, to prove Proposition 3.15] it is enough to show that if $t \in\{2,3\}$ and

$$
P=\sum_{i=1}^{4} a_{i} u_{i}+\sum_{i=1}^{4} b_{i} v_{i}+\sum_{i=1}^{4} c_{i} w_{i} \in S_{c l}^{c}
$$


(with $a_{i}, b_{i}, c_{i} \in k$ ) are such that there exists $h \in k^{*}$ with $g^{t} P=h * P$, then $P=0$, where by definition

$$
h * P=\sum_{i=1}^{4} h a_{i} u_{i}+\sum_{i=1}^{4} h b_{i} v_{i}+\sum_{i=1}^{4} h^{2} c_{i} w_{i} .
$$

Step 1. We first study the action of $g^{2}$. The action of $g^{2}$ on the direct sum of $V_{i}$ can be described by

$$
g^{2}=\left(u_{1}, u_{3}, u_{2}\right)\left(v_{1}, v_{3}, v_{2}\right)\left(u_{4}\right)\left(v_{4}\right)\left(w_{1}, w_{3}, w_{2}\right)\left(w_{4}\right)
$$

in the sense that $g^{2} u_{1}=u_{3}, g^{2} u_{3}=u_{2}, g^{2} u_{2}=u_{1}$, etc. We assume

$$
g^{2}(P)=h * P
$$

for some nonzero $P$ as in (3.10), and we will get a contradiction. Since $P$ is nonzero, by Lemma 3.9 there exists $i$, with $1 \leq i \leq 4$, such that $a_{i} \neq 0$ or $b_{i} \neq 0$. Since the eigenvalues of $g^{2}$ acting on any of $V_{1}, \ldots, V_{4}$ are contained in the set $\left\{1, \zeta^{2}, \zeta^{4}\right\}$, we get that $h \in\left\{1, \zeta^{2}, \zeta^{4}\right\}$.

Step 2. We assume that $h=1$ in (3.11), and we will get a contradiction. By looking at the action of $g^{2}$ on $V_{1}$ and $V_{2}$ we have

$$
a_{3}=a_{2}=a_{1}, \quad b_{3}=b_{2}=b_{1} .
$$

Using the equations $h_{1}, h_{2}, h_{3}$ we additionally get

$$
b_{1}=b_{2}=b_{3}=b_{4}=0, \quad a_{4}=-3 r_{5} a_{1} .
$$

Substituting to $m_{4}^{s}$ we get $\left(a_{1}\right)^{3} r_{1}=0$. Hence $a_{1}=0$ (since $r_{1}$ is general), which implies that all $a_{j}=0$ and all $b_{j}=0$, contradicting Lemma 3.9.

Step 3. We assume that $h=\zeta^{2}$ in (3.11), and we will get a contradiction. By looking at the action of $g^{2}$ on each $V_{i}$ we have

$$
\begin{aligned}
& a_{3}=\zeta^{2} a_{1}, \quad a_{2}=\zeta^{4} a_{1}, \quad b_{3}=\zeta^{2} b_{1}, \quad b_{2}=\zeta^{4} b_{1}, \\
& a_{4}=b_{4}=c_{4}=0, \quad c_{3}=\zeta^{4} c_{1}, \quad c_{2}=\zeta^{2} c_{1} .
\end{aligned}
$$

Substituting to $l_{1}^{s}$ we get $a_{1} c_{1}=0$, and substituting to $m_{1}^{s}$ we get $b_{1} c_{1}=0$. We can assume that $c_{1}=0$; otherwise we get that all $a_{j}=0$ and all $b_{j}=0$, contradicting Lemma 3.9. As a consequence, $c_{i}=0$ for $1 \leq i \leq 4$.

Substituting to $l_{4}^{s}$ we get

$$
3 r_{2}\left(a_{1}\right)^{2} b_{1}+r_{4}\left(b_{1}\right)^{3}=0
$$

while substituting to $m_{4}^{s}$ we get

$$
r_{1}\left(a_{1}\right)^{3}+3 r_{3} a_{1}\left(b_{1}\right)^{2}=0 .
$$

It is clear that for general values of the $r_{i}$ the last two equations have no nonzero common solutions for $a_{1}$ and $b_{1}$, so we get all $a_{j}=0$ and all $b_{j}=0$, contradicting $P$ nonzero.

Step 4. We assume that $h=\zeta^{4}$ in (3.11), and we will get a contradiction. By looking at the action of $g^{4}$ on each $V_{i}$ we have

$$
\begin{aligned}
& a_{3}=\zeta^{4} a_{1}, \quad a_{2}=\zeta^{2} a_{1}, \quad b_{3}=\zeta^{4} b_{1}, \quad b_{2}=\zeta^{2} b_{1}, \\
& a_{4}=b_{4}=c_{4}=0, \quad c_{3}=\zeta^{2} c_{1}, \quad c_{2}=\zeta^{4} c_{1} .
\end{aligned}
$$


Substituting to $l_{1}^{s}$ we get $a_{1} c_{1}=0$, and substituting to $m_{1}^{s}$ we get $b_{1} c_{1}=0$. We can assume that $c_{1}=0$; otherwise we get that all $a_{j}=0$ and all $b_{j}=0$, contradicting Lemma 3.9. As a consequence, $c_{i}=0$ for $1 \leq i \leq 4$.

Substituting to $l_{4}^{s}$ we get

$$
3 r_{2}\left(a_{1}\right)^{2} b_{1}+r_{4}\left(b_{1}\right)^{3}=0
$$

while substituting to $m_{4}^{s}$ we get

$$
3 r_{1}\left(a_{1}\right)^{3}+r_{2} a_{1}\left(b_{1}\right)^{2}=0 .
$$

It is clear that for general values of the $r_{i}$ the last two equations have no nonzero common solutions for $a_{1}$ and $b_{1}$, so we again get all $a_{j}=0$ and all $b_{j}=0$, contradicting $P$ nonzero.

Step 5. We now study the action of $g^{3}$. The action of $g^{3}$ on the direct sum of the $V_{i}$ can be described by

$$
g^{3}\left(u_{i}\right)=-u_{i}, \quad g^{3}\left(v_{i}\right)=v_{i}, \quad g^{3}\left(w_{i}\right)=-w_{i}
$$

for $1 \leq i \leq 4$.

We assume

$$
g^{3}(P)=h * P
$$

for some nonzero $P$ as in (3.10), and we will get a contradiction. Arguing as in Step 1 , we get $h \in\{1,-1\}$.

Step 6. We assume that $h=1$ in (3.12), and we will get a contradiction. Indeed, by the way $g^{3}$ acts we have $a_{i}=c_{i}=0$ for $1 \leq i \leq 4$. Substituting to $e_{14}^{s}, e_{24}^{s}, e_{34}^{s}$ we get respectively

$$
b_{2} b_{3}=b_{1} b_{3}=b_{1} b_{2}=0
$$

hence at least two of the three $b_{1}, b_{2}, b_{3}$ are 0 . Using $h_{1}$ and $h_{2}$ we get that $b_{i}=0$ for $1 \leq i \leq 4$, contradicting $P$ nonzero.

Step 7. We assume that $h=-1$ in (3.12), and we will get a contradiction. Indeed, by the way $g^{3}$ acts we have $b_{i}=c_{i}=0$ for $1 \leq i \leq 4$. Fix $1 \leq i<j \leq 4$. Substituting to $e_{i j}^{s}$ we get that $a_{p} a_{q}=0$, where the indices $p, q$ have the property $\{p, q, i, j\}=\{1,2,3,4\}$. As a consequence at least three of the four $a_{i}$ are 0 . Using $h_{3}$ we get that all $a_{i}$ are 0 , contradicting $P$ nonzero. This finishes the proof of Proposition 3.14.

\section{Final Remarks And QUestions}

Remark 4.1. In [CR, Corti and Reid pose the problem of interpreting the Gorenstein formats arising from unprojection as solutions to universal problems. What can be said about the generic $\left(\begin{array}{c}n \\ 2\end{array}\right)$ Pfaffians ideal of Definition 2.2?

Remark 4.2. During the proof of Theorem 3.16, we established that the étale 6 to 1 numerical Campedelli covers of our construction have trivial algebraic fundamental group. We expect that they also have trivial topological fundamental group, but we were unable to prove it.

Remark 4.3. We believe that the ideas of the present paper can also be useful for the study of the numerical Campedelli surfaces with torsion groups $\mathbb{Z} / 2$ and $\mathbb{Z} / 3$. 
Consider first the $\mathbb{Z} / 3$ torsion case. The numeric invariants suggest that the étale 3 to 1 cover of such a numerical Campedelli surface could be a suitable member of $\left|-2 K_{V_{3}}\right|$, where

$$
V_{3} \subset \mathbb{P}\left(1^{2}, 2^{7}, 3^{5}\right)
$$

is a (candidate) codimension 10 Fano 3 -fold having a basket of ten $1 / 2(1,1,1)$ singularities, which appears in Brown's online database of the graded ring $[\mathrm{Br}]$. Moreover, $\mathrm{Br}$ suggests that $V_{3}$ can, perhaps, be constructed as a result of a series of symmetric looking type II unprojections (cf. [R1], [P3]).

Similarly, the numeric invariants for the $\mathbb{Z} / 2$ torsion case suggests that the étale 2 to 1 cover of such a numerical Campedelli could be a suitable member of $\left|-2 K_{V_{2}}\right|$, where

$$
V_{2} \subset \mathbb{P}\left(1,2^{6}, 3^{8}\right)
$$

is a (candidate) codimension 11 Fano 3 -fold having a basket of twelve $1 / 2(1,1,1)$ singularities and also appearing in $\left[\mathrm{Br}\right.$. Moreover, $[\mathrm{Br}]$ suggests that $V_{2}$ can, perhaps, be constructed as a result of a series of again symmetric looking type IV unprojections (cf. [R3]).

\section{ACKNOWLEDGMENTS}

The authors wish to thank Miles Reid and Margarida Mendes Lopes for important discussions and suggestions which have improved the presentation of the paper. The authors are also thankful to an anonymous referee for suggesting improvements to the presentation of the paper and a remark that helped us find the moduli bound. The authors are grateful for the financial support of Fundação Gulbenkian. The second author was supported by the Portuguese Fundação para a Ciência e a Tecnologia through Grant SFRH/BPD/22846/2005 of POCI2010/FEDER.

\section{REFERENCES}

[BH] Bruns, W. and Herzog, J., Cohen-Macaulay rings. Revised edition, Cambridge Studies in Advanced Mathematics 39, CUP 1998 MR1251956 (95h:13020)

[BPHV] Barth, W., Hulek, K., Peters, C., Van de Ven, A., Compact complex surfaces. Second enlarged edition, Ergebnisse der Mathematik und ihrer Grenzgebiete, 4, Springer, 2004 MR2030225 (2004m:14070)

[Br] Brown, G., Graded ring database homepage, online searchable database, available from http://pcmat12.kent.ac.uk/grdb/index.php

[BV] Bruns, W. and Vetter, U., Determinantal rings. Lecture Notes in Math. 1327, Springer 1988 MR953963 (89i:13001)

[CR] Corti, A. and Reid, M., Weighted Grassmannians, in Algebraic geometry, A volume in memory of Paolo Francia, M. Beltrametti et al. (eds.), de Gruyter 2002, 141-163 MR.1954062 (2003m:14076)

[Do] Dolgachev, I, Weighted projective varieties in Group actions and vector fields, 34-71, Lecture Notes in Math., 956, Springer 1982 MR704986 (85g:14060)

[Ei] Eisenbud, D., Commutative algebra, with a view toward algebraic geometry. Graduate Texts in Mathematics, 150. Springer-Verlag, 1995 MR1322960 (97a:13001)

[FOV] Flenner, H., O'Carrol, L. and Vogel, W., Joins and intersections. Springer Monographs in Mathematics. Springer-Verlag, 1999 MR 1724388 (2001b:14010)

[Fr] Frantzen, Kr., On K3-surfaces in weighted projective space. Univ. of Warwick M.Sc. thesis, Sep 2004 v+55 pp., available from http://pcmat12.kent.ac.uk/grdb/Doc/papers.php

[GPS01] Greuel, G.-M, Pfister, G., and Schönemann, H., Singular 2.0. A Computer Algebra System for Polynomial Computations. Centre for Computer Algebra, University of Kaiserslautern (2001), available from http://www.singular.uni-kl.de

[Ha] Hartshorne, R., Algebraic Geometry. Graduate Texts in Mathematics, 52. SpringerVerlag, 1977 MR0463157 (57:3116) 
[IF] Iano-Fletcher, A., Working with weighted complete intersections, in Explicit birational geometry of 3-folds, 101-173, London Math. Soc. Lecture Note Ser., 281, CUP 2000 MR.1798982 (2001k:14089)

[KM] Kustin, A. and Miller, M., Constructing big Gorenstein ideals from small ones, J. Algebra 85 (1983), 303-322 MR725084 (85f:13014)

[LP] Lee, Y. and Park, J., A simply connected surface of general type with $p_{g}=0$ and $K^{2}=2$, Invent. Math. 170 (2007), no. 3, 483-505 MR2357500

[L] Liu, Q., Algebraic geometry and arithmetic curves, Oxford Graduate Texts in Mathematics, 6. Oxford University Press, 2002 MR.1917232 (2003g:14001)

[MP] Mendes Lopes, M. and Pardini, R., Numerical Campedelli surfaces with fundamental group of order 9, J. Eur. Math. Soc. 10 (2008), no. 2, 457-476 MR2390332

[Na] Naie, D., Numerical Campedelli surfaces cannot have the symmetric group as the algebraic fundamental group, J. London Math. Soc. 59 (1999), 813-827 MR1709082 (2000f:14055)

[P1] Papadakis, S., Gorenstein rings and Kustin-Miller unprojection, Univ. of Warwick Ph.D. thesis, Aug 2001, vi + 72 pp., available from http://www.math.ist.utl.pt/ papadak/

[P2] Papadakis, S., Kustin-Miller unprojection with complexes, J. Algebraic Geometry 13 (2004), 249-268 MR2047698(2005d:13025)

[P3] Papadakis, S., Type II unprojection, J. Algebraic Geometry 15 (2006), 399-414 MR.2219843 (2007c:14051)

[PR] Papadakis, S. and Reid, M., Kustin-Miller unprojection without complexes, J. Algebraic Geometry 13 (2004), 563-577 MR2047681(2005j:14068)

[R1] Reid, M., Graded Rings and Birational Geometry, in Proc. of algebraic symposium (Kinosaki, Oct 2000), K. Ohno (Ed.) 1-72, available from www.maths.warwick.ac.uk/ $\sim$ miles/3folds

[R2] Reid, M., Campedelli versus Godeaux, in Problems in the theory of surfaces and their classification (Cortona, 1988), 309-365, Sympos. Math., XXXII, Academic Press, London, 1991 MR 1273384 (95h:14031)

[R3] Reid, M., Examples of type IV unprojection, preprint, math.AG/0108037, 16 pp.

Centre for Mathematics, University of Coimbra, 3001-454 Coimbra, Portugal

E-mail address: neves@mat.uc.pt

Center for Mathematical Analysis, Geometry, and Dynamical Systems, Departamento de Matemática, Instituto Superior Técnico, Universidade Técnica de Lisboa, 1049-001 Lisboa, Portugal

E-mail address: papadak@math.ist.utl.pt 